Research Article

\title{
The Effect of Caching on CP and ISP Policies in Information-Centric Networks
}

\author{
Hamid Garmani $\left(\mathbb{D}\right.$, Driss Ait Omar ${ }^{(D)}$, Mohamed El Amrani $\mathbb{D}$, Mohamed Baslam (iD, \\ and Mostafa Jourhmane
} Information Processing and Decision Support Laboratory, Faculty of Sciences and Technics, Sultan Moulay Slimane University,
Beni Mellal, Morocco

Correspondence should be addressed to Hamid Garmani; garmani.hamid@gmail.com

Received 25 June 2020; Revised 2 October 2020; Accepted 14 October 2020; Published 30 October 2020

Academic Editor: Ramon Aguero

Copyright (C) 2020 Hamid Garmani et al. This is an open access article distributed under the Creative Commons Attribution License, which permits unrestricted use, distribution, and reproduction in any medium, provided the original work is properly cited.

\begin{abstract}
Internet traffic volume is increasing, and this causes scalability issues in content delivery. Information-centric network has been introduced to support this increase in Internet traffic through caching. While collaborative caching in information-centric network is a crucial feature to improve network performance and reduce delivery costs in content distribution, the current pricing strategies on the Internet are not incentive compatible with information-centric network interconnection. In this paper, we focus on the economic incentive interactions in caching deployment between several types of information-centric network providers (content provider and Internet service provider). In particular, we develop game-theoretic models to study the interaction between providers in an information-centric network model where the providers are motivated to cache and share content. We use a generalized Zipf distribution to model content popularity. We formulate the interactions between the Internet service providers and between the content providers as a noncooperative game. We use a Stackelberg game model to capture the interactions between the content provider and Internet service providers. Through mathematical analysis, we prove the existence and uniqueness of the Nash equilibrium under some conditions. An iterative and distributed algorithm based on best response dynamics is proposed to achieve the equilibrium point. The numerical simulations illustrate that our proposed game models result in a win-win solution.
\end{abstract}

\section{Introduction}

The growth of Internet traffic data is rapidly increasing due to the explosion of sites on the Internet such as YouTube, Dailymotion, and Netflix. Many technologies have been proposed to control the large volume of information, improve the spectrum efficiency, and increase network capacity. Besides traditional technologies, information-centric network (ICN) has drawn researchers' attention in recent years, which is a new communication paradigm to increase the efficiency of content delivery. The main ideas in ICNs are the following: (1) content is located by name instead of by IP, and (2) every ICN node can cache and serve the requested content. The advantages motivating the ICN approach are scalable, persistent, security, mobility, etc [1].
It is considered that network caching can get many advantages to end-users, the Internet service provider (ISP), and the content providers (CPs). For end-users, caching content in ISP cache can minimize the delay in getting content, which enhances the quality of experience. From the viewpoint of the ISPs, network caching can lower the traffic outgoing to the other ISPs or CPs, which reduce the backhaul bandwidth consumption. So, literature studies [2-4] suggest that ISPs should deploy a cache with capacity. Furthermore, for $\mathrm{CPs}$, content requests can be satisfied from the ISP cache, which can lower the load on the CPs, economize backhaul bandwidth resources, and avert network congestions. More importantly, when the $\mathrm{CP}$ is temporarily offline, the ISP cache can continue to provide 
content to end-users, which improves the robustness of the network.

Inspired by the above discussion, ICN has many technical benefits; however, ICN has so far stayed only in the research literature, compared to CDN or HTTP caches that have a massive deployment. The reason is that the vast providers (ISPs and CPs) need economic incentives in addition to benefits of technic to collaborate in the deployment of the ICN. This paper aims at designing a flexible economic model to prompt the deployment of ICN.

In our previous work [5], we study the interaction among two ISPs in ICN, where each ISP controls the amount of content cached and adjusts their prices and its quality of service. In this paper, we extend our previous studies by considering a general framework for incentive CPs and ISPs to collaborate in caching and distributing content in ICN. We consider a hierarchical network model with multiple CPs and multiple ISPs. We use a game-theoretic approach to study the interaction between CPs and ISPs.

Our main contributions are summarized as follows:

(i) We formulate the interactions among ISPs based on four market parameters, network access price, caching, cache access price, and quality of service. In addition, we formulate the competition among CPs as a function of two parameters, content access price and credibility of content. The interaction between providers is analyzed as a noncooperative game.

(ii) We use the Stackelberg game to analyze competition among CPs and ISPs, and we adopt the method backward induction to solve the proposed Stackelberg game.

(iii) We analytically prove the existence and uniqueness of the Nash equilibrium.

(iv) Through simulation, the interactions between model parameter and provider strategies are represented. Also, the impacts of model parameter on provider revenue are revealed. We finally carry out that the ISPs, the CPs, and end-users can receive benefit from caching investment.

The rest of this paper is organized as follows. Section 2 discusses related work. In Section 3, we describe the system model. In Section 4, we formulate and analyze the noncooperative game. In Section 5, we model a Stackelberg game. Then, we present the numerical results in Section 6. Conclusions are provided in Section 7.

\section{Related Work}

The existing literature has studied several problems related to caching in the small cell network, cloud radio access networks, and ISPs. In [6], the authors have used the meanfield game to study the problem of distributed caching in a dense wireless small cell network. In [7], the authors proposed a joint network slicing and content caching for inband wireless backhaul-based virtualized wireless networks, where requested contents can be retrieved from either the associated base stations or the close content cached at the edge of the core network. The authors in [8] propose an ICNbased caching approach for videos in $5 \mathrm{G}$ networks, which considers both the mobility of users and the popularity of videos. In [9], the authors designed a proactive caching policy for a HetNet scenario to jointly optimize the choice of which files to store at the mobile users and the small base stations. The authors in [10] studied distributed cooperative hybrid caching problems based on content-awareness in D2D networks. In [11], the authors proposed a dynamic Stackelberg-Cournot game in which multiple mobile network operators offer payable storage service to multiple CPs competing for caching space. The authors in [12] proposed an edge caching scheme for vehicular content networks to provide vehicular content delivery. An analytical model is developed, which takes account of vehicle velocity, road traffic, and content popularity for characterizing the content distribution at the edge of vehicular content networks. In [13], the authors have studied caching with asynchronous requests and presented a coded joint pushing and caching method to determine the packets to be pushed and cached. The authors in [14] proposed a new cooperative edge caching architecture for content-centric 5G networks, where mobile edge computing resources are utilized for enhancing edge caching capability. They show that the proposed scheme minimizes content access latency and improves caching resource utilization. The authors in [15] presented an interdomain cache-sharing market routing technique based on the bargaining game, which took full advantage of the cache resource to extend cache sharing among domains to Internet service providers.

The second research line [3, 16-19] attracts more attention compared to the first one, as how to incentivize network players to collaborate in the deployment of the ICN. The authors in [20] explored the interdomain routing policies in ICN. They argued that the network caching will influence the business relationships between neighboring autonomous systems. The authors in [19] model the economic incentive independencies between ISPs and CP and propose a reward-based incentive caching model for content caching and sharing in ICN, where the CP decides the reward price of caching each content and ISPs decide which content to be cached. They used a Stackelberg game model to capture the interactions between ISP and CP. In [21], the authors formalized the profit of ISPs when implementing content-centric networking and investigate the likelihood of content-centric networking spreading through numerical evaluation. The authors in [22] analyzed the economic interactions in one ISP and proposed a policy to make ICN economically profitable for ISPs. In [18], the authors proposed a new incentive mechanism that improves both the ISPs' and CPs' profits via the use of auction theory. In the proposed model, the ISP earns profits from caching by alleviating traffic load on transit links and participating in content selling, where the contents are from multiple CPs. The authors in [23] proposed a new incentive mechanism for paid content caching that satisfies both ISP and CPs through the use of a reverse auction. The authors in [3] have modeled the economic interactions between the different actors of an information-centric network, using the framework of game 
theory. They show the uniqueness of the Nash equilibrium in the pricing caching game. The authors of this work show that caching is profitable. The authors in [24] proposed a pricing model to establish an economic incentive mechanism for caching and sharing content in ICN that consists of an ISP, a transit ISP, and a CP. In [25], the authors investigate a noncooperative game between an ISP and a CP, where the ISP changes its caching strategy, and the CP can control its pricing strategy. They considered that the polarity of the side-payment (from the ISP to the CP) in an ICN is different from that in the current Internet model (i.e., host-centric communication model). In [16], the authors modeled the content popularity by the generalized Zipf distribution and used the noncooperative game to decide caching and pricing strategies in information-centric network (ICN). In [26], the authors investigated an economical pricing approach to address caching resource management issues in the 5G wireless networks. They used Cournot, Stackelberg, and Bertrand models to study this model. The authors in [27] analyzed the impact of different factors on the incentives of related players in ICN, including the ISPs, CDNs, and CPs. The authors built a simple model to address the economic incentive problem of different ICN network players in a hierarchical caching infrastructure.

All these related works mentioned above economics of service pricing in the ICN, which consists of an ISP and a CP. Their model restricts the network to one CP and few ISPs. Our work belongs to the second research line but differs from these existing works in the following five aspects:

(1) We developed an analytical framework for the distribution of popular content in ICN, which comprises multiple CPs, multiple ISPs, and a set of endusers.

(2) We model and analyze the interplay between ISPs as a function of four market parameters: network access price, quality of services, caching, and cache access price.

(3) We model and analyze the interplay between CPs as a function of four market parameters: content access price, the credibility of content, caching cost, and transmission price.

(4) We study a game between ISPs and among CPs as a noncooperative game. The analyzed economic interaction in our model is not involved by the existing works.

(5) We analytically prove the existence of equilibrium between CPs and between ISPs, and then we applied the best response algorithm for learning Nash equilibrium. In addition, we use the price of anarchy to evaluate the performance of the system at the Nash equilibrium.

\section{Problem Modeling}

The system model is illustrated in Figure 1, with $\mathscr{F}$ ISPs, $N$ $\mathrm{CPs}$, and several end-users. These entities have diverse roles in ICN, the CPs produce content, the ISP provides access service or connects end-users, and end-users consume content. The strategies of each CP consists of four parts, the price for delivering content to end-users, the price for selling content to ISPs, the price for forwarding content trough ISP, and the credibility of content, which is a function of the quality of service and quality of content. Each ISP can cache the entire or portion of all content provided by CPs. Also, each ISP chooses five market parameters: network access price, the price to access to content in the cache, caching, the credibility of content in the cache, and the quality of service. A detailed summary of notations is presented in Table 1.

3.1. Content Popularity. Let $H$ be the number of items provided by CPs, and each item has a measure of popularity reflected by the probability of requests for it. We consider a model where the popularity of content is the same for all users. As in previous works (e.g., [28-30]), in this paper, the probability of requests follows generalized Zipf distribution function as follows:

$$
\phi_{h}=A^{-1} h^{-\eta},
$$

where $A=\sum_{h=1}^{H} h^{-\eta}, h^{\eta}$ is the rank of item $h-$ th, and $\eta$ is the skewness of the popularity distribution. The item ranked in the order of their popularity where the item $h$ is the $h$ most popular item, i.e., $h=1$ is the most popular item and $h=H$ is the least popular item.

3.2. Demand Model. $D_{i j}$ is the average demand of end-users who connect to the $\mathrm{CP}_{i}$ through the ISP ${ }_{j}$ which depends on content access price $p_{c_{i}}$, credibility $c_{i}$, network access price $p_{s_{i}}$, QoS $q_{s_{i}}$, and cache access price $p_{c c_{j}}$ (see [31-33]). The demand $D_{i j}$ depends also on prices $\mathbf{p}_{c_{-i}}$, credibilities $\mathbf{c}_{-i}$, prices $\mathbf{p}_{s_{-j}}$, prices $\mathbf{p}_{c c_{-j}}$, and QoS set by the opponents. Eventually, $D_{i j}$ is decreasing with respect to $p_{c_{i}}, p_{s_{i}}$, and $p_{c c_{j}}$ and increasing with respect to $p_{c_{n}}, n \neq i, p_{s_{f}}, p_{c c_{f}}$, and $f \neq j$, whereas it is increasing with respect to $c_{i}$ and $q_{s_{i}}$ and decreasing with respect to $c_{n}, n \neq i, q_{s_{f}}$, and $f \neq j$. Then, the demand functions $D_{i j}$ can be written as follows:

$$
\begin{aligned}
D_{i j}= & d_{i j}-\alpha_{j}^{j} p_{s_{j}}+\beta_{j}^{j} q_{s_{j}}-\rho_{j}^{j} p_{c c_{j}}-\delta_{i}^{i} p_{c_{i}}+\sigma_{i}^{i} c_{i} \\
& -\sum_{f=1, f \neq j}^{F}\left(\alpha_{j}^{f} p_{s_{f}}-\beta_{j}^{f} q_{s_{j}}+\rho_{j}^{f} p_{c c_{f}}\right) \\
& +\sum_{n=1, n \neq i}^{N}\left(\delta_{i}^{n} p_{c_{n}}-\sigma_{i}^{n} c_{n}\right),
\end{aligned}
$$

where $d_{i j}, \alpha_{j}^{f}, \beta_{j}^{f}, \rho_{i}^{f}, \delta_{i}^{n}$, and $\sigma_{i}^{n}$ are the positive constant. The parameter $d_{i j}$ reflects the total potential demand of endusers. $\delta_{i}^{n}$ and $\sigma_{i}^{n}$ denote the responsiveness of demand $D_{i j}$ to price $p_{c_{n}}$ and credibility $c_{n}$ of $\mathrm{CP}_{n} . \alpha_{j}^{f}, \beta_{j}^{f}$, and $\rho_{j}^{f}$ denote the responsiveness of demand $D_{i j}$ to price $p_{s_{f}}$, price $p_{c c_{f}}$, and QoS $q_{s_{f}}$ of $\mathrm{ISP}_{f}$.

Assumption 1. The sensitivity $\alpha$ verifies:

$$
\alpha_{j}^{j} \geq \sum_{f=1, f \neq j}^{F} \alpha_{j}^{f}, \quad \forall j=1, \ldots, F .
$$




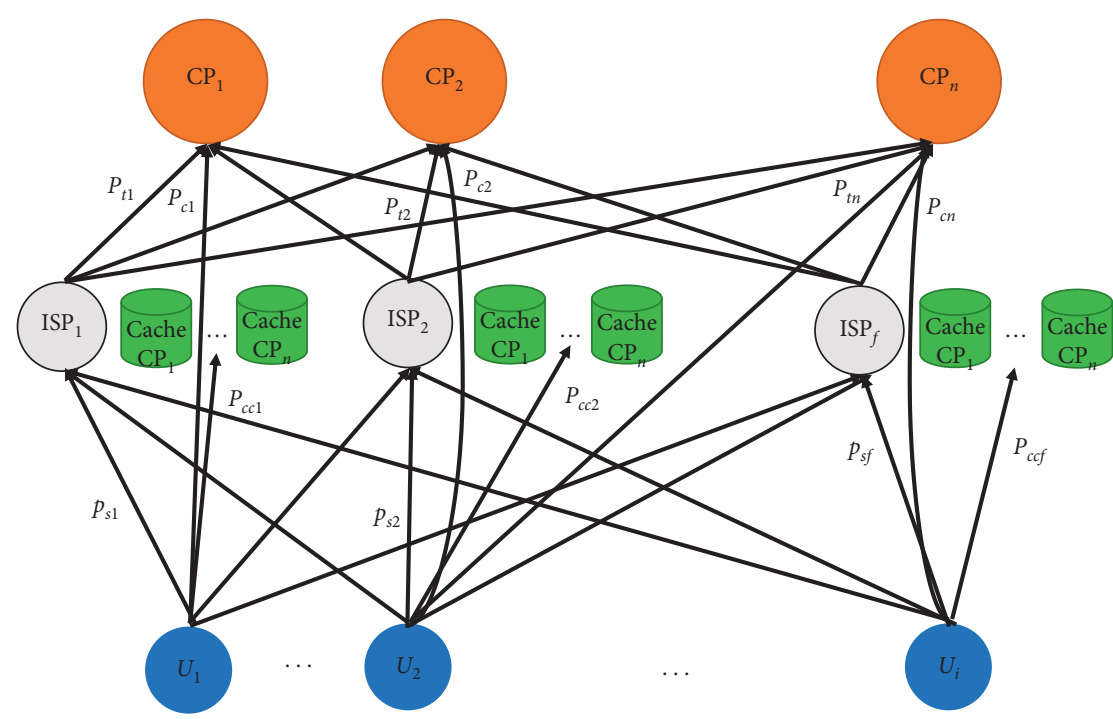

FIGURE 1: Interaction between different providers in a simplified model of ICN.

TABLE 1: Summary of notation.

\begin{tabular}{|c|c|}
\hline Notation & Description \\
\hline$N$ & Number of CPs \\
\hline$H$ & Number of items that the CP sells \\
\hline$F$ & Number of ISPs \\
\hline$p_{s_{j}}$ & Network access price of ISP $_{j}$ \\
\hline$P_{c c_{j}}$ & Price to access to the content in the cache of $\mathrm{ISP}_{j}$ \\
\hline$q_{s_{j}}$ & Quality of service of ISP $_{j}$ \\
\hline$c_{j}$ & Credibility of content of ISP ${ }_{j}$ \\
\hline$C_{i h}$ & Caching cost of item $h$ provided by $\mathrm{CP}_{i}$ \\
\hline$v_{j}$ & Backhaul bandwidth cost \\
\hline$p_{c_{i}}$ & Price to access to content of $\mathrm{CP}_{i}$ \\
\hline$c_{i}$ & Credibility of content of $\mathrm{CP}_{i}$ \\
\hline$p_{t_{\dot{p}}}$ & Transmission fee of $\mathrm{CP}_{i}$ \\
\hline$\alpha_{j_{f}}^{f^{\prime}}$ & Sensitivity of ISP ${ }_{j}$ to price $p_{s_{f}}$ of ISP $_{f}$ \\
\hline$\beta_{j}^{f}$ & Sensitivity of $\mathrm{ISP}_{j}$ to QoS $q_{s_{f}}$ of $\operatorname{ISP}_{f}$ \\
\hline$\rho_{j}^{f}$ & Sensitivity of ISP ${ }_{j}$ to price $p_{c c_{f}}$ of ISP ${ }_{f}$ \\
\hline$\delta_{i}^{n}$ & Sensitivity of $\mathrm{CP}_{i}$ to price $p_{c_{n}}$ of $\mathrm{CP}_{n}$ \\
\hline$\sigma_{i}^{n}$ & Sensitivity of $\mathrm{CP}_{i}$ to credibility of content $c_{n}$ of $\mathrm{CP}_{n}$ \\
\hline$d_{i j}$ & The total potential demand of end-users \\
\hline$D_{i j}$ & 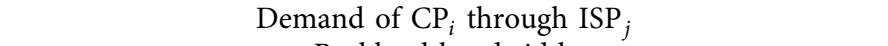 \\
\hline$B_{j}$ & Backhaul bandwidth \\
\hline$h^{\eta}$ & The rank of item $h$ \\
\hline$\eta$ & The skewness of the popularity distribution \\
\hline$\lambda$ & The sensitivity of the credibility of content to the quality of service \\
\hline$\mu$ & The sensitivity of the credibility of content to the quality of content \\
\hline$\theta_{i}$ & The cost to produce a unit of credibility $c_{i}$ \\
\hline
\end{tabular}

The sensitivity $\beta$ verifies:

$$
\beta_{j}^{j} \geq \sum_{f=1, f \neq j}^{F} \beta_{j}^{f}, \quad \forall j=1, \ldots, F .
$$

The sensitivity $\rho$ verifies:

$$
\rho_{j}^{j} \geq \sum_{f=1, f \neq j}^{F} \rho_{j}^{f}, \quad \forall j=1, \ldots, F .
$$

The sensitivity $\delta$ verifies:

$$
\delta_{i}^{i} \geq \sum_{n=1, n \neq i}^{N} \delta_{i}^{n}, \quad \forall i=1, \ldots, N .
$$

The sensitivity $\sigma$ verifies:

$$
\sigma_{i}^{i} \geq \sum_{n=1, n \neq i}^{N} \sigma_{i}^{n}, \quad \forall i=1, \ldots, N .
$$


Assumption 1 will be needed to ensure the uniqueness of Nash equilibrium.

3.3. Utility Model of the ISP. The utility of the $\mathrm{ISP}_{j}$ is equal to revenue minus cost:

$$
\begin{aligned}
U_{\mathrm{ISP}_{j}}= & p_{s_{j}} \sum_{i=1}^{N} D_{i j}+\sum_{i=1}^{N} \sum_{h=1}^{H} \phi_{h}\left\{\tau_{j}^{i} k_{i j h} D_{i j}\left(p_{c c_{j}}-C_{i h}\right)\right. \\
& \left.-p_{t_{i}} D_{i j} c_{i}\left(1-\tau_{j}^{i} k_{i j h}\right)\right\} \\
& -v_{j} B_{j} \sum_{i=1}^{N}\left(H-\tau_{i}^{j} \sum_{h=1}^{H} k_{i j h}\right)-\theta_{j} c_{j}^{2} .
\end{aligned}
$$

$p_{s} \sum_{i=1}^{N} D_{i j}$ is the revenue of network access. $C_{i h}$ is the caching cost of the item $h$ provided by $\mathrm{CP}_{i} . \tau_{j}^{i}$ is the proportion of demand for content produced by $\mathrm{CP}_{i}$ and served by $\operatorname{ISP}_{j}$ cache, which can be expressed as $\tau_{j}^{i}=\max \left\{1, c_{j} / c_{i}\right\}$. $\tau_{j}^{i} D_{i j}$ is the demand served by $\operatorname{ISP}_{j}$ cache. $\phi_{h} \tau_{j}^{i} k_{i j h} D_{i j}\left(p_{c c_{j}}-\right.$ $\left.C_{i h}\right)$ is the revenue that results when the $\operatorname{ISP}_{j}$ serves the requested demand $\phi_{h} \tau_{j}^{i} k_{i j h} D_{i j}$ of the item $h$ from its cache. In ICN models, the ISP pays to the CP the transmission fees ([25]). $\phi_{h} p_{t_{i}} D_{i j} c_{i}\left(1-\tau_{j}^{i} k_{i j h}\right)$ is the transmission fee that the $\mathrm{ISP}_{j}$ pays to the $\mathrm{CP}_{i}$, when the $\mathrm{CP}_{i}$ forwards the request demand $\phi_{h} D_{i j}\left(1-\tau_{j}^{i} k_{i j h}\right)$ of the item $h$ through the $\operatorname{ISP}_{j} . \theta_{j}$ is the cost to produce a unit of the credibility of content $c_{j}$. $\theta_{j} c_{j}^{2}$ is the cost to produce credibility of content $c_{j} . v_{j}$ is the unit backhaul bandwidth cost paid by $\operatorname{ISP}_{j} . B_{j}$ is the backhaul bandwidth needed to serve demand $\sum_{i=1}^{N} D_{i j}$. $B_{j} \sum_{i=1}^{N}\left(H-\tau_{i}^{j} \sum_{h=1}^{H} k_{i j h}\right)$ is the backhaul bandwidth needed for serving the demand $\sum_{i=1}^{N} D_{i j}\left(H-\tau_{i}^{j} \sum_{h=1}^{H} k_{i j h}\right) . B_{j}$ is the backhaul bandwidth required by the $\operatorname{ISP}_{j}$. The quality of service $q_{s_{j}}$ is defined as the expected delay (see [34]):

$$
q_{s_{j}}=\frac{1}{\sqrt{\text { Delay }}}=\sqrt{B_{j}-\sum_{i=1}^{N} D_{i j}}
$$

which means that

$$
B_{j}=q_{s_{j}}^{2}+\sum_{i=1}^{N} D_{i j} .
$$

Then, the utility of the $\mathrm{ISP}_{j}$ is given by

$$
\begin{aligned}
U_{\mathrm{ISP}_{j}}= & p_{s_{j}} \sum_{i=1}^{N} D_{i j}+\sum_{i=1}^{N} \sum_{h=1}^{H} \phi_{h}\left\{\tau_{j}^{i} k_{i j h} D_{i j}\left(p_{c c_{j}}-C_{i h}\right)\right. \\
& \left.-p_{t_{i}} D_{i j} c_{i}\left(1-\tau_{j}^{i} k_{i j h}\right)\right\} \\
& -v_{j}\left(q_{s_{j}}^{2}+\sum_{i=1}^{N} D_{i j}\right)\left(H N-\sum_{i=1}^{N} \tau_{i}^{j} \sum_{h=1}^{H} k_{i j h}\right)-\theta_{j} c_{j}^{2} .
\end{aligned}
$$

$k_{i j h}=1$ means that the ISP $_{j}$ decides to cache the item $h$ of the $\mathrm{CP}_{i}$, because the revenue of $\mathrm{ISP}_{j}$ by serving the requested demand of the item $h$ from their cache is larger than the cost for forwarding the request demand of the item $h$ to the $\mathrm{CP}_{i} \cdot k_{i j h}=0$ means that the $\mathrm{ISP}_{j}$ does not cache the item $h$ of $\mathrm{CP}_{i}$, because the cost for forwarding the request demand of the item $h$ to the $\mathrm{CP}_{i}$ is larger than the revenue of ISP $_{j}$ by serving the requested demand of the item $h$ from their cache.

3.4. Utility Model of the CP. The utility of $\mathrm{CP}_{i}$ is equal to revenue minus cost:

$$
\begin{aligned}
U_{\mathrm{CP}_{i}}= & \sum_{j=1}^{F} \sum_{h=1}^{H} \phi_{h}\left\{\left(p_{c_{i}}+p_{t_{i}} c_{i}\right) D_{i j}\left(1-\tau_{j}^{i} k_{i j h}\right)+\tau_{j}^{i} k_{i j h} C_{i h} D_{i j}\right\} \\
& -\theta_{i} c_{i}^{2} .
\end{aligned}
$$

$p_{c_{i}} \phi_{h} \sum_{j} D_{i j}\left(1-\tau_{j}^{i} k_{i j h}\right)$ is the revenue of $\mathrm{CP}_{i}$ by serving the request demand $\phi_{h} D_{i j}\left(1-\tau_{j}^{i} k_{i j h}\right)$ of item $h . p_{t_{i}}$ is the transmission fee that $\mathrm{CP}_{i}$ charges to ISPs. $p_{t_{i}} \phi_{h} D_{i j}\left(1-\tau_{j}^{i} k_{i j h}\right)$ is the transmission revenue of the $\mathrm{CP}_{i}$, when $\mathrm{CP}_{i}$ forwards the request demand of item $h$ through ISP $_{j} \cdot \tau_{j}^{i} k_{i j h} C_{i h} D_{i j}$ is the profit of $\mathrm{CP}_{i}$ when the demand $\tau_{j}^{i} k_{i j h} D_{i j}$ are satisfied from the ISP ${ }_{j}$ cache. $\theta_{i} c_{i}^{2}$ is the cost to produce the credibility of content $c_{i}$. The credibility of content $c_{i}$ of $\mathrm{CP}_{i}$ is a function of the quality of service $q_{s s_{i}}$ and quality content $q_{c_{i}}$, which can be expressed as [33, 35-37]

$$
c_{i}=\lambda q_{s s_{i}}+\mu q_{c_{i}}
$$

where $\lambda$ and $\mu$ are the two positive constants, which represent, respectively, the sensitivity of the credibility of content to QoS and QoC. We define the QoS as the "expected delay," which is computed by the Kleinrock function (see [34]). The quality of content QoC can be specified for a specific domain of content, e.g., quality of video streaming.

Then, the utility of $\mathrm{CP}_{i}$ is given by

$$
\begin{aligned}
U_{\mathrm{CP}_{i}}= & \sum_{j=1}^{F} \sum_{h=1}^{H} \phi_{h}\left\{\left(p_{c_{i}}+p_{t_{i}}\left(\lambda q_{s s_{i}}+\mu q_{c_{i}}\right)\right) D_{i j}\left(1-\tau_{j}^{i} k_{i j h}\right)\right. \\
& \left.+\tau_{j}^{i} k_{i j h} C_{i h} D_{i j}\right\}-\theta_{i}\left(\lambda q_{s s_{i}}+\mu q_{c_{i}}\right)^{2} .
\end{aligned}
$$

\section{Game Analysis}

Let $G_{1}=\left[\mathscr{F},\left\{P_{s_{j}}, P_{c c_{j}}, Q_{s_{j}}\right\},\left\{U_{\text {ISP }_{j}}().\right\}\right]$ denote the noncooperative price QoS price game (NPQPG), where $\mathscr{F}=\{1, \ldots, F\}$ is the set of ISPs, $P_{s_{j}}$ is the strategy set of price, $P_{c c_{j}}$ is the strategy set of price, $Q_{s_{j}}$ is the strategy set of QoS, and $U_{\mathrm{ISP}_{j}}($.$) is the utility function of \mathrm{ISP}_{j}$ defined in equation (11). We assume that the strategy spaces $P_{s_{j}}, P_{c c_{j}}$, and $Q_{s_{j}}$ of each ISPs are compact and convex sets with maximum and minimum constraints. Thus, for each ISP ${ }_{j}$, we consider as respective strategy spaces the closed intervals: $P_{s_{j}}=\left[\underline{p}_{s_{j}}, \bar{p}_{s_{j}}\right], P_{c c_{j}}=\left[\underline{p}_{c c_{j}}, \bar{p}_{c c_{j}}\right]$, and $Q_{s_{j}}=\left[\underline{q}_{s_{j}}, \bar{q}_{s_{j}}\right]$. Let, the 
price vector $\mathbf{p}_{s}=\left(p_{s_{1}}, \ldots, p_{s_{F}}\right)^{T} \in P_{s}^{F}=P_{s_{1}} \times P_{s_{2}} \times \cdots \times P_{s_{F}}$, the price vector $\mathbf{p}_{c c}=\left(p_{c_{1}}, \ldots, p_{c_{F}}\right)^{T} \in P_{c c}^{F}=P_{c_{1}} \times P_{c_{2}} \times$ $\cdots \times P_{c_{F}}$, and the QoS vector $\mathbf{q}_{s}=\left(q_{s_{1}}, \ldots, q_{s_{F}}\right)^{T^{2}} \epsilon$ $Q_{s}^{F}=Q_{s_{1}} \times Q_{s_{2}} \times \cdots \times Q_{s_{F}}$.

Let $G_{2}=\left[\mathcal{N},\left\{P_{c_{i}}, Q_{s s_{i}}, Q_{c_{i}}\right\},\left\{U_{\mathrm{CP}_{i}}().\right\}\right]$ denote the noncooperative QoS price QoC game (NQPQG), where $\mathcal{N}=$ $\{1, \ldots, N\}$ is the set of CPs, $P_{c_{i}}$ is the strategy set of price, $Q_{s s_{i}}$ is the strategy set of QoS, $Q_{c_{i}}$ is the strategy set of QoC, and $U_{\mathrm{CP}_{i}}$ (.) is the utility function of $\mathrm{CP}_{i}$ defined in equation (14). We assume that the strategy spaces $P_{c_{i}}, Q_{s s_{i}}$, and $Q_{c_{i}}$ of each $\mathrm{CP}$ are compact and convex sets with maximum and minimum constraints. Thus, for each $\mathrm{CP}_{i}$, we consider as respective strategy spaces the closed intervals: $P_{c_{i}}=\left[\underline{p}_{c_{i}}, \bar{p}_{c_{i}}\right]$, $Q_{s s_{i}}=\left[\underline{q}_{s s_{i}}, \bar{q}_{s s_{i}}\right]$, and $Q_{c_{i}}=\left[\underline{q}_{c_{i}}, \bar{q}_{c_{i}}\right]$. Let, the price vector $\mathbf{p}_{c}=\left(p_{c_{1}}, \ldots, p_{c_{N}}\right)^{T} \in P_{c}^{N}=\bar{P}_{c_{1}} \times P_{c_{2}} \times \cdots \times P_{c_{N}}$, the QoS vector $\mathbf{q}_{s s}=\left(q_{s s_{1}}, \ldots, q_{s s_{N}}\right)^{T} \in Q_{s s}^{N}=Q_{s s_{1}} \times Q_{s s_{2}} \times \cdots \times Q_{s s_{N}}$, and the QoC vector $\mathbf{q}_{c}=\left(q_{c_{1}}, \ldots, q_{c_{N}}\right)^{T} \in$ $Q_{c}^{N}=Q_{c_{1}} \times Q_{c_{2}} \times \cdots \times Q_{c_{N}}$.

4.1. Price $P_{s}$ Game. A NPQPG in price is defined for fixed $\mathbf{p}_{c c} \in P_{c c}, \quad \mathbf{q}_{s} \in Q_{s} \quad$ as $\quad G_{1}\left(\mathbf{p}_{c c}, \mathbf{q}_{s}\right)=\left[\mathscr{F}\left\{P_{s_{j}}\right\},\left\{U_{\mathrm{ISP}_{j}}\right.\right.$ $\left.\left(., \mathbf{p}_{c c}, \mathbf{q}_{s}\right\}\right]$.

Definition 1. A price vector $\mathbf{p}_{s}^{*}=\left(p_{s_{1}}^{*}, \ldots, p_{s_{F}}^{*}\right)$ is a Nash equilibrium of the NPQPG $G_{1}\left(\mathbf{p}_{c c}, \mathbf{q}_{s}\right)$ if

$$
\begin{gathered}
\forall\left(j, p_{s_{j}}\right) \in\left(\mathscr{F}, P_{s_{j}}\right) \\
U_{\text {ISP }_{j}}\left(p_{s_{j}}^{*}, \mathbf{p}_{s_{-j}}^{*}, \mathbf{p}_{c c}, \mathbf{q}_{s}\right) \geq U_{\text {ISP }_{j}}\left(p_{s_{j}}, \mathbf{p}_{s_{-j}}^{*}, \mathbf{p}_{c c}, \mathbf{q}_{s}\right) .
\end{gathered}
$$

Theorem 1. For each $\mathbf{p}_{c c} \in P_{c c}, \quad \mathbf{q}_{s} \in Q_{s}$, the game $\left[\mathscr{F},\left\{P_{s_{j}}\right\},\left\{U_{I S P_{j}}\left(., \mathbf{p}_{c c}, \mathbf{q}_{s}\right\}\right]\right.$ admits a unique Nash equilibrium.

Proof. The game $\mathrm{G}_{1}\left(\mathbf{P}_{\mathrm{cc}}, \mathbf{q}_{\mathrm{s}}\right)$ admits at least one equilibrium, if and only if its second derivative of the utility $U_{\text {ISP }}$ function with respect to price Psi is nonpositive. $p_{s_{j}}$ is nonpositive. Indeed, we first compute the first derivative of $U_{\mathrm{ISP}_{j}}$ :

$$
\begin{aligned}
\frac{\partial U_{\mathrm{ISP}_{j}}}{\partial p_{s_{j}}}= & -N \alpha_{j}^{j} p_{s_{j}}+\sum_{i=1}^{N} D_{i j} \\
& +\sum_{i=1}^{N} \sum_{h=1}^{H} \phi_{h}\left\{-\alpha_{j}^{j} \tau_{j}^{i} k_{i j h}\left(p_{c c_{j}}-C_{i h}\right)\right. \\
& \left.+\alpha_{j}^{j} p_{t_{i}} c_{i}\left(1-\tau_{j}^{i} k_{i j h}\right)\right\} \\
& +v_{j} N \alpha_{j}^{j}\left(H N-\tau_{j}^{i} \sum_{h=1}^{H} k_{i j h}\right) .
\end{aligned}
$$

Then, we calculate the second derivative:

$$
\frac{\partial^{2} U_{\text {ISP }_{j}}}{\partial p_{s_{j}}^{2}}=-2 \alpha_{j}^{j} N \leq 0
$$

The second derivative of the utility function is always nonpositive, then the utility function is concave, which ensures the existence of a Nash equilibrium.

We use the following proposition that holds for a concave game [38]: if a concave game satisfies the dominance solvability condition

$$
-\frac{\partial^{2} U_{\mathrm{ISP}_{j}}}{\partial p_{s_{j}}^{2}} \geq \sum_{f=1, f \neq j}^{F}\left|\frac{\partial^{2} U_{\mathrm{ISP}_{j}}}{\partial p_{s_{j}} \partial p_{s_{f}}}\right|
$$

then the game $G_{1}\left(\mathbf{p}_{c c}, \mathbf{q}_{s}\right)$ admits a unique Nash equilibrium point.

The mixed partial is written as

$$
\frac{\partial^{2} U_{\mathrm{ISP}_{j}}}{\partial p_{s_{j}} \partial p_{s_{f}}}=\alpha_{j}^{f} N
$$

Then,

$$
-\frac{\partial^{2} U_{\text {ISP }_{j}}}{\partial p_{s_{j}}^{2}}-\sum_{f=1, f \neq j}^{F}\left|\frac{\partial^{2} U_{\text {ISP }_{j}}}{\partial p_{s_{j}} \partial p_{s_{f}}}\right|=N\left(2 \alpha_{j}^{j}-\sum_{f=1, f \neq j}^{F} \alpha_{j}^{f}\right) \geq 0 .
$$
point.

Thus, the $G_{1}\left(\mathbf{p}_{c c}, \mathbf{q}_{s}\right)$ admits a unique Nash equilibrium 4.2. Price $P_{c c}$ Game. A NPQPG in price is defined for fixed $\mathbf{p}_{s} \in P_{s}, \quad \mathbf{q}_{s} \in Q_{s} \quad$ as $\quad G_{1}\left(\mathbf{p}_{s}, \mathbf{q}_{s}\right)=\left[\mathscr{F},\left\{P_{c c_{j}}\right\},\left\{U_{\mathrm{ISP}_{j}}\right.\right.$ $\left.\left(\mathbf{p}_{s}, ., \mathbf{q}_{s}\right\}\right]$.

Definition 2. A price vector $\mathbf{p}_{c c}^{*}=\left(p_{c c_{1}}^{*}, \ldots, p_{c c_{F}}^{*}\right)$ is a Nash equilibrium of the NPQPG $G_{1}\left(\mathbf{p}_{s}, \mathbf{q}_{s}\right)$ if

$$
\begin{aligned}
\forall\left(j, p_{c c_{j}}\right) & \in\left(\mathscr{F}, P_{c c_{j}}\right), \quad U_{\mathrm{ISP}_{j}}\left(\mathbf{p}_{s}, p_{c c_{j}}^{*}, \mathbf{p}_{c c_{-j}}^{*}, \mathbf{q}_{s}\right) \\
& \geq U_{\mathrm{ISP}_{j}}\left(\mathbf{p}_{s}, p_{c c_{j}}, \mathbf{p}_{c c_{-j}}^{*}, \mathbf{q}_{s}\right) .
\end{aligned}
$$

Theorem 2. For each $\mathbf{p}_{s} \in P_{s}, \quad \mathbf{q}_{s} \in Q_{s}$, the game $\left[\mathscr{F},\left\{P_{c c_{j}}\right\},\left\{U_{I S P_{j}}\left(\mathbf{p}_{s}, ., \mathbf{q}_{s}\right\}\right]\right.$ admits a unique Nash equilibrium.

The proof of the above theorem can be found in Appendix A.

4.3. $Q o S Q_{s}$ Game. An NPQPG in QoS is defined for fixed $\mathbf{p}_{s} \in P_{s}, \quad \mathbf{p}_{c c} \in P_{c c} \quad$ as $G_{1}\left(\mathbf{p}_{s}, \mathbf{p}_{c c}\right)=\left[\mathscr{F},\left\{Q_{s_{j}}\right\},\left\{U_{\mathrm{ISP}_{j}}\left(\mathbf{p}_{s}\right.\right.\right.$, $\left.\left.\mathbf{p}_{c c},.\right\}\right]$.

Definition 3. A QoS vector $\mathbf{q}_{s}^{*}=\left(q_{s_{1}}^{*}, \ldots, q_{s_{F}}^{*}\right)$ is a Nash equilibrium of the NPQPG $G_{1}\left(\mathbf{p}_{s}, \mathbf{p}_{c c}\right)$ if 


$$
\begin{gathered}
\forall\left(j, q_{s_{j}}\right) \in\left(\mathscr{F}, Q_{s_{j}}\right), \\
U_{\mathrm{ISP}_{j}}\left(\mathbf{p}_{s}, \mathbf{p}_{c c}, q_{s_{j}}^{*}, \mathbf{q}_{s_{-j}}^{*}\right) \geq U_{\mathrm{ISP}_{j}}\left(\mathbf{p}_{s}, \mathbf{p}_{c c}, q_{s_{j}}, \mathbf{q}_{s_{-j}}^{*}\right) .
\end{gathered}
$$

Theorem 3. For each $\mathbf{p}_{s} \in P_{s}, \quad \mathbf{p}_{c c} \in P_{c c}$, the game $\left[\mathscr{F},\left\{Q_{s_{j}}\right\},\left\{U_{I S P_{j}}\left(\mathbf{p}_{s}, \mathbf{p}_{c c}, \cdot\right\}\right]\right.$ admits a unique Nash equilibrium. $B$.

The proof of the above theorem can be found in Appendix

4.4. Price $P_{c}$ Game. An NQPQG in price $p_{c}$ is defined for fixed $\mathbf{q}_{s s} \in Q_{s s}, \quad \mathbf{q}_{c} \in Q_{c}$ as $G_{2}\left(\mathbf{q}_{s s}, \mathbf{q}_{c}\right)=\left[\mathcal{N},\left\{P_{c_{i}}\right\},\left\{U_{\mathrm{CP}_{i}}\right.\right.$ $\left.\left.\left(., \mathbf{q}_{s s}, \mathbf{q}_{c}\right)\right\}\right]$.

Definition 4. A price vector $\mathbf{p}_{c}^{*}=\left(p_{c_{1}}^{*}, \ldots, p_{c_{N}}^{*}\right)$ is a Nash equilibrium of the NQPQG $G_{2}\left(\mathbf{q}_{s s}, \mathbf{q}_{c}\right)$ if

$$
\begin{gathered}
\forall\left(i, p_{c_{i}}\right) \in\left(\mathcal{N}, P_{c_{i}}\right), \\
U_{\mathrm{CP}_{i}}\left(p_{c_{i}}^{*}, \mathbf{p}_{c_{-i}}^{*}, \mathbf{q}_{s}, \mathbf{q}_{c}\right) \geq U_{\mathrm{CP}_{i}}\left(p_{c_{i}}, \mathbf{p}_{c_{-i}}^{*}, \mathbf{q}_{s}, \mathbf{q}_{c}\right) .
\end{gathered}
$$

Theorem 4. For each $\mathbf{q}_{s s} \in Q_{s s}, \quad \mathbf{q}_{c} \in Q_{c}$, the game $\left[\mathcal{N},\left\{P_{c_{i}}\right\},\left\{U_{C P_{i}}\left(., \mathbf{q}_{s s}, \mathbf{q}_{c}\right)\right\}\right]$ admits a unique Nash equilibrium. C.

The proof of the above theorem can be found in Appendix

4.5. QoCQ $Q_{c}$ Game. An NQPQG in QoC is defined for a fixed $\mathbf{p}_{c} \in P_{c}, \quad \mathbf{q}_{s s} \in Q_{s s} \quad$ as $\quad G_{2}\left(\mathbf{p}_{c}, \mathbf{q}_{s s}\right)=\left[\mathcal{N},\left\{Q_{c_{i}}\right\},\left\{U_{\mathrm{CP}_{i}}\right.\right.$ $\left.\left.\left(\mathbf{p}_{c}, \mathbf{q}_{s s},.\right)\right\}\right]$.

Definition 5. A QoC vector $\mathbf{q}_{c}^{*}=\left(q_{c_{1}}^{*}, \ldots, q_{c_{N}}^{*}\right)$ is a Nash equilibrium of the NQPQG $G_{2}\left(\mathbf{p}_{c}, \mathbf{q}_{s s}\right)$ if

$$
\begin{gathered}
\forall\left(i, q_{c_{i}}\right) \in\left(\mathcal{N}, Q_{c_{i}}\right), \\
U_{\mathrm{CP}_{i}}\left(\mathbf{p}_{c}, \mathbf{q}_{s s}, q_{c_{i}}^{*}, \mathbf{q}_{c_{-i}}^{*}\right) \geq U_{\mathrm{CP}_{i}}\left(\mathbf{p}_{c}, \mathbf{q}_{s s}, q_{c_{i}}, \mathbf{q}_{c_{-i}}^{*}\right) .
\end{gathered}
$$

Theorem 5. For each $\mathbf{p}_{c} \in P_{c}, \quad \mathbf{q}_{s s} \in Q_{s s}$, the game $\left[\mathcal{N},\left\{Q_{c_{i}}\right\},\left\{U_{C P_{i}}\left(\mathbf{p}_{c}, \mathbf{q}_{s s},.\right)\right\}\right]$ admits a unique Nash equilibrium. $D$.

The proof of the above theorem can be found in Appendix

4.6. QoS $Q_{s s}$ Game. An NQPQG in QoS is defined for a fixed $\mathbf{p}_{c} \in P_{c}, \mathbf{q}_{c} \in Q_{c}$ as $G_{2}\left(\mathbf{p}_{c}, \mathbf{q}_{c}\right)=\left[\mathcal{N},\left\{Q_{s s_{i}}\right\},\left\{U_{\mathrm{CP}_{i}}\left(\mathbf{p}_{c}, ., \mathbf{q}_{c}\right)\right\}\right]$.

Definition 6. A QoS vector $\mathbf{q}_{s s}^{*}=\left(q_{s s_{1}}^{*}, \ldots, q_{s s_{N}}^{*}\right)$ is a Nash equilibrium of the NQPQG $G_{2}\left(\mathbf{p}_{c}, \mathbf{q}_{c}\right)$ if

$$
\begin{gathered}
\forall\left(i, q_{s s_{i}}\right) \in\left(\mathcal{N}, Q_{s s_{i}}\right), \\
U_{\mathrm{CP}_{i}}\left(\mathbf{p}_{c}, q_{s s_{i}}^{*}, \mathbf{q}_{s s_{-i}}^{*}, \mathbf{q}_{c}\right) \geq U_{\mathrm{CP}_{i}}\left(\mathbf{p}_{c}, q_{s s_{i}}, \mathbf{q}_{s s_{-i}}^{*}, \mathbf{q}_{c}\right) .
\end{gathered}
$$

Theorem 6. For each $\mathbf{p}_{c} \in P_{c}, \mathbf{q}_{c} \in Q_{c}$, the game $\left[\mathcal{N},\left\{Q_{s s_{i}}\right\},\left\{U_{C P_{i}}\left(\mathbf{p}_{c}, ., \mathbf{q}_{c}\right)\right\}\right]$ admits a unique Nash equilibrium.

4.7. Learning Nash Equilibrium. The idea of best response is useful when we are trying to find an approach to reach a steady-state of a game, i.e., a Nash equilibrium of a game. The best response dynamics scheme consists of a sequence of rounds, where in each round after the first, each provider observes the policy taken by its competitors in previous rounds and inputs them in its decision process to update its policy. In the first round, the choice of each provider is the best response based on its arbitrary belief about what the other players will choose.

Algorithm 1 summarizes the best response learning steps that each provider has to perform to find the Nash equilibrium.

Such as

(i) $E$ denotes CP or ISP

(ii) $\mathcal{O}$ refers to $\mathscr{N}$ or $\mathscr{K}$

(iii) $x$ refers to the vector price $p_{c}$, vector price $p_{s}$, vector price $p_{c c}$, vector $q_{s}$, vector $q_{c}$ or vector $q_{s s}$

(iv) $X_{o}$ refers to the policy profile price, QoS or QoC

The proof of the above theorem can be found in Appendix E.

4.8. Price of Anarchy. The price of anarchy (PoA) is defined as the ratio between the performance measures of the worst equilibrium and the optimal outcome. A PoA close to 1 indicates that the equilibrium is approximately socially optimal, and thus, the consequences of selfish behavior are benign.

In [39], we measure the loss of efficiency due to actors' selfishness as the quotient between the social welfare obtained at the Nash equilibrium and the maximum value of the social welfare:

$$
\text { PoA }=\frac{\min _{p_{s}, p_{c c}, q_{s}, p_{c}, q_{c}, q_{s s}} W_{\mathrm{NE}}\left(p_{s}, p_{c c}, q_{s}, p_{c}, q_{c}, q_{s s}\right)}{\max _{p_{s}, p_{c c}, q_{s}, p_{c}, q_{c}, q_{s s}} W\left(p_{s}, p_{c c}, q_{s}, p_{c}, q_{c}, q_{s s}\right)}
$$

where $\quad W\left(p_{s}, p_{c c}, q_{s}, p_{c}, q_{c}, q_{s s}\right)=\sum_{j=1}^{M} U_{i}\left(p_{s}, p_{c c}, q_{s}, p_{c}\right.$, $\left.q_{c}, q_{s s}\right)$ is the social welfare function and $W_{\mathrm{NE}}\left(p_{s}^{*}, p_{c c}^{*}, q_{s}^{*}, p_{c}^{*}, q_{c}^{*}, q_{s s}^{*}\right)=\sum_{j=1}^{M} U_{i}\left(p_{s}, p_{c c}, q_{s}, p_{c}, q_{c}, q_{s s}\right)$ is a sum of utilities of all actors at Nash equilibrium.

\section{Stackelberg Game Formulation}

The Stackelberg game is an extension of the noncooperative game with a bilevel hierarchy. The Stackelberg game models a game between two groups of players, namely, leaders and followers. The leaders have the privilege of acting the first while the followers work according to the leaders' actions. Backward induction is always utilized to solve the Stackelberg game. In the backward induction method, the followers' optimal strategies are solved firstly, given the leader strategies. Secondly, leader players' optimal strategies are 
(1) Initialize vectors $x(0)=\left[x_{1}(0), \ldots, x_{O}(0)\right]$ randomly;

(2) For each $E_{o}, o \in \mathcal{O}$ at time instant $t$ computes:

(a) $x_{o}(t+1)=\operatorname{argmax}_{x_{0} \in X_{0}}\left(U_{E_{0}}(x(t))\right)$.

(3) If $\forall o \in \mathcal{O},\left|x_{j}(t+1)-x_{j}(t)\right|<\varepsilon$, then STOP.

(4) Else, $t \longleftarrow t+1$ and go to step (2)

Algorithm 1: Best response algorithm.

solved. At the first stage of the Stackelberg game, the CP sets its QoS and QoC. At the second stage of the game, given the QoS and QoC of the CP, the ISP sets the QoS and QoC of content in its cache. The proposed Stackelberg game modeling is shown in Figure 2.

Firstly, we consider the second stage game. Given the QoS and QoC of the CP, the ISP needs to determine the optimal QoS and QoC to maximize this payoff.

Proposition 1. The optimal QoS and QoC of the ISPs is given as follows:

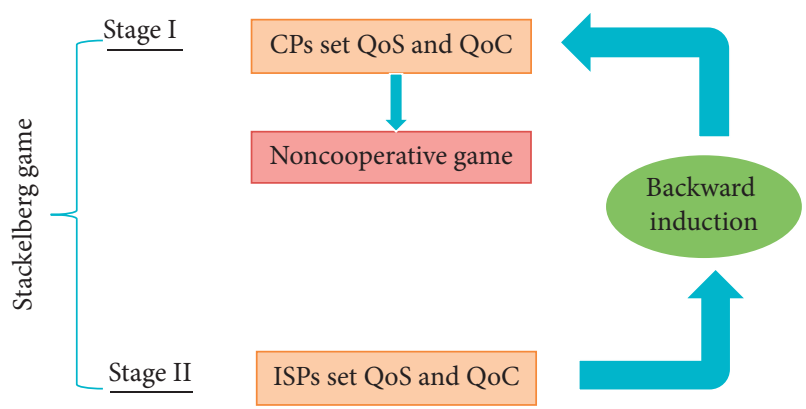

Figure 2: Stackelberg game modeling.

$$
\begin{aligned}
& q_{c_{j}}=\sum_{i=1}^{N} \sum_{h=1}^{H} \phi_{h}\left\{\frac{k_{i j h} D_{i j}\left(p_{c c_{j}}-C_{i h}\right)}{2 \mu \theta_{j} c_{i}}+\frac{p_{t_{i}} D_{i j} k_{i j h}}{2 \mu \theta_{j}}\right\}+\frac{v_{j} B_{j} \sum_{h=1}^{H} k_{i j h}}{2 \mu \theta_{j} c_{i}}-\frac{\lambda q_{s s_{j}}}{\mu}, \\
& q_{s s_{j}}=\sum_{i=1}^{N} \sum_{h=1}^{H} \phi_{h}\left\{\frac{k_{i j h} D_{i j}\left(p_{c c_{j}}-C_{i h}\right)}{2 \lambda \theta_{j} c_{i}}+\frac{p_{t_{i}} D_{i j} k_{i j h}}{2 \lambda \theta_{j}}\right\}+\frac{v_{j} B_{j} \sum_{h=1}^{H} k_{i j h}}{2 \lambda \theta_{j} c_{i}}-\frac{\mu q_{c_{j}}}{\lambda} .
\end{aligned}
$$

Proof. the optimal condition for QoS and QoC is to let the

On the other hand, we have first-order differentiation equal to 0 as follows:

$$
\begin{gathered}
\frac{\partial U_{\mathrm{ISP}_{j}}}{\partial q_{c_{j}}}=0, \\
\frac{\partial U_{\mathrm{ISP}_{j}}}{\partial q_{s s_{j}}}=0 .
\end{gathered}
$$

$$
\begin{aligned}
& \frac{\partial U_{\mathrm{ISP}_{j}}}{\partial q_{c_{j}}}=\sum_{i=1}^{N} \sum_{h=1}^{H} \phi_{h}\left\{\frac{\mu k_{i j h} D_{i j}\left(p_{c c_{j}}-C_{i h}\right)}{c_{i}}+\mu p_{t_{i}} D_{i j} k_{i j h}\right\}+\frac{\mu v_{j} B_{j} \sum_{h=1}^{H} k_{i j h}}{c_{i}}-2 \mu \theta_{j}\left(\lambda q_{s s_{j}}+\mu q_{c_{j}}\right), \\
& \frac{\partial U_{\mathrm{ISP}_{j}}}{\partial q_{s s_{j}}}=\sum_{i=1}^{N} \sum_{h=1}^{H} \phi_{h}\left\{\frac{\lambda k_{i j h} D_{i j}\left(p_{c c_{j}}-C_{i h}\right)}{c_{i}}+\lambda p_{t_{i}} D_{i j} k_{i j h}\right\}+\frac{\lambda v_{j} B_{j} \sum_{h=1}^{H} k_{i j h}}{c_{i}}-2 \lambda \theta_{j}\left(\lambda q_{s s_{j}}+\mu q_{c_{j}}\right) .
\end{aligned}
$$


Table 2: Parameter setting used for numerical examples.

\begin{tabular}{|c|c|c|c|c|c|c|}
\hline$\alpha_{1}^{1}=\alpha_{2}^{2}$ & $\alpha_{j}^{f}, j \neq f$ & $\beta_{1}^{1}=\beta_{2}^{2}$ & $\beta_{j}^{f}, j \neq f$ & $\rho_{1}^{1}=\rho_{2}^{2}$ & $\rho_{j}^{f}, j \neq f$ & $d_{11}$ \\
\hline 0.55 & 0.45 & 0.55 & 0.45 & 0.55 & 0.45 & 250 \\
\hline $\begin{array}{l}\sigma_{1}^{1}=\sigma_{2}^{2} \\
0.55\end{array}$ & $\begin{array}{c}\sigma_{i}^{n}, i \neq n \\
0.45\end{array}$ & $\begin{array}{c}\delta_{1}^{1}=\delta_{2}^{2} \\
10\end{array}$ & $\begin{array}{c}\delta_{i}^{n}, i \neq n \\
0.5\end{array}$ & $p_{t_{1}}=p_{10}$ & $\begin{array}{c}\theta_{i}, i \in\{1,2\} \\
2\end{array}$ & $\begin{array}{l}d_{12} \\
250\end{array}$ \\
\hline $\begin{array}{l}\bar{p}_{c_{1}}=\bar{p}_{c_{2}} \\
1000\end{array}$ & $\underline{p}_{c_{1}}=\underline{p}_{c_{2}}$ & $\begin{array}{c}\bar{q}_{s s_{1}}=\bar{q}_{s s_{2}} \\
1000\end{array}$ & $\underline{q}_{s s_{1}}=\underline{q}_{1}{\underline{s s_{2}}}$ & $\begin{array}{c}\bar{q}_{c_{1}}=\bar{q}_{c_{2}} \\
1000\end{array}$ & $\underline{q}_{c_{1}}=\underline{q}_{c_{2}}$ & $\begin{array}{l}d_{21} \\
200\end{array}$ \\
\hline $\begin{array}{l}\bar{p}_{s_{1}}=\bar{p}_{s_{2}} \\
1000\end{array}$ & $\underline{p}_{s_{1}}=\underline{p}_{s_{2}}$ & $\begin{array}{c}\bar{q}_{s_{1}}=\bar{q}_{s_{2}} \\
1000\end{array}$ & $\underline{q}_{s_{1}}=\underline{q}_{s_{2}}$ & $\begin{array}{c}\bar{p}_{c c_{1}}=\bar{p}_{c c_{2}} \\
1000\end{array}$ & $\underline{p}_{c c_{1}}=\underline{p}_{1} c_{c_{2}}$ & $\begin{array}{l}d_{22} \\
250\end{array}$ \\
\hline$v_{1}=v_{2}$ & $H$ & $C_{1 h}=C_{2 h}, \forall h$ & $A$ & $\eta$ & $\theta_{j}, j \in\{1,2\}$ & $\lambda=\mu$ \\
\hline 10 & 40 & 40 & 10 & 0.5 & 10 & 0.5 \\
\hline
\end{tabular}

Then, the QoS and QoC that maximize the ISPs' profits are given as follows:

$$
\begin{aligned}
q_{c_{j}} & =\sum_{i=1}^{N} \sum_{h=1}^{H} \phi_{h}\left\{\frac{k_{i j h} D_{i j}\left(p_{c c_{j}}-C_{i h}\right)}{2 \mu \theta_{j} c_{i}}+\frac{p_{t_{i}} D_{i j} k_{i j h}}{2 \mu \theta_{j}}\right\}+\frac{v_{j} B_{j} \sum_{h=1}^{H} k_{i j h}}{2 \mu \theta_{j} c_{i}}-\frac{\lambda q_{s s_{j}}}{\mu}, \\
q_{s s_{j}} & =\sum_{i=1}^{N} \sum_{h=1}^{H} \phi_{h}\left\{\frac{k_{i j h} D_{i j}\left(p_{c c_{j}}-C_{i h}\right)}{2 \lambda \theta_{j} c_{i}}+\frac{p_{t_{i}} D_{i j} k_{i j h}}{2 \lambda \theta_{j}}\right\}+\frac{v_{j} B_{j} \sum_{h=1}^{H} k_{i j h}}{2 \lambda \theta_{j} c_{i}}-\frac{\mu q_{c_{j}}}{\lambda} .
\end{aligned}
$$

Secondly, we solve the first stage of the Stackelberg game, where the CPs set QoS and QoC. We formulate the game between $\mathrm{CPs}$ as a noncooperative game. The noncooperative game is described in the above Sections 4.5 and 4.6.

\section{Numerical Investigation}

In this section, we provide the simulation results to illustrate the competitive interactions in the ICN market. Additionally, we will present a detailed description of the impact of the system parameters on the provider policies. Furthermore, we will discuss the effect of the caching cost and the number of cached items on the price of anarchy. To simplify the complex market environment, we consider a network scenario that includes two ISPs and two CPs. Simulation settings are listed in Table 2.

Figures 3-8 show the convergence toward Nash equilibrium price, Nash equilibrium QoS, and Nash equilibrium QoC of all providers. Figures 3-8 demonstrate the existence and uniqueness of a Nash equilibrium point at which no providers can profitably deviate given the strategies of another opponent.

Figure 9 shows the price $p_{c}$ of the CP under different cost C. From Figure 9, we can observe that price $p_{c}$ of the designed model is decreasing as cost $C$ increases. The reason is that a higher cost generates a larger profit from caching, which makes CPs decrease their price $p_{c}$ to induce increased demand from the end-users.

Figure 10 illustrates the Nash equilibrium price $p_{s}$ with caching cost $C$ for two ISPs. Figure 10 shows that as the caching cost $C$ increases, the ISPs set a greater price $p_{s}$. The reason is that as the caching cost $C$ becomes expensive, the
ISPs do not have any motivation to cache content. Hence, the ISPs forward more requests of content to the CPs, which increases the transmission fee and the cost of backhaul bandwidth. Then, the ISPs are obliged to increase their price $p_{s}$ to compensate for the cost of transmission and the cost of backhaul bandwidth.

Figure 11 shows the price $p_{c c}$ under different content popularities. From Figure 11, we can observe that $p_{c c}$ is decreasing as content popularity increases. The reason is that a larger Zipf skewness parameter generates a larger proportion of content requests for the popular contents, which makes more requests directly satisfied by the ISP caches and then brings a higher profit.

The effect of the number of cached items $H$ on QoS and QoC is shown in Figures 12 and 13. QoS and QoC of the proposed model are growing as $H$ increases because a more significant number of cached items can make more content cached and more requests of end-users satisfied by the ISP cache. As a result, the revenue of CPs increases because the revenue from caching increases. Therefore, the CPs increase their QoS and QoC to induce increased demand from endusers.

Figure 14 illustrates the Nash equilibrium price with the number of cached items $H$ for two ISPs. As $H$ increases, the number of cached item increases. When the number of cached item increases, the proportion of content requests of ISP cache increases, the transmission cost decreases, and the cost of backhaul bandwidth decreases, which brings a higher profit. Therefore, the ISPs decrease their price $p_{s}$ to induce increased demand from the end-users.

Figure 15 shows the ISP PoA variation curve as a function of caching cost $C$. In that figure, PoA increases with 


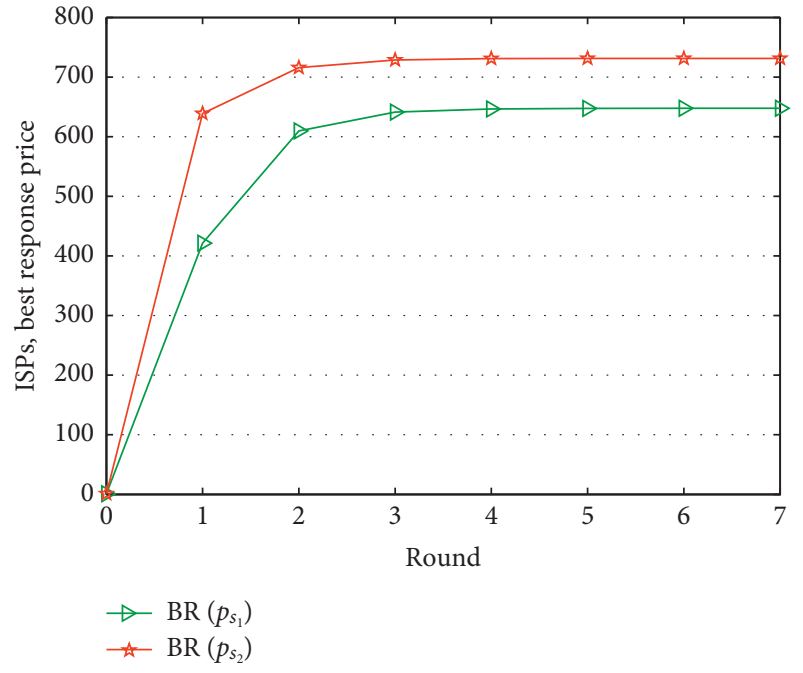

FIGURE 3: Nash equilibrium price under the best response dynamic.

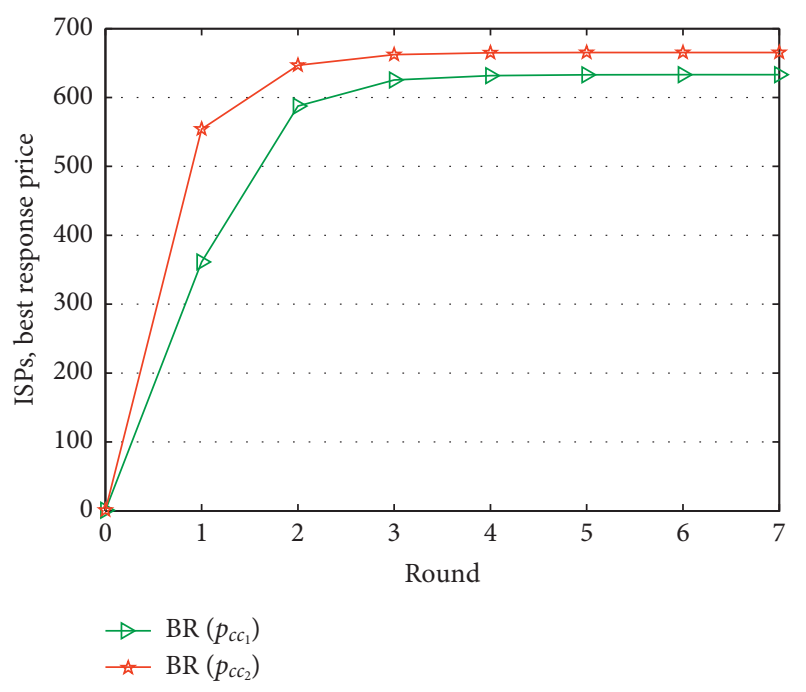

FIGURE 4: Nash equilibrium price under the best response dynamic.

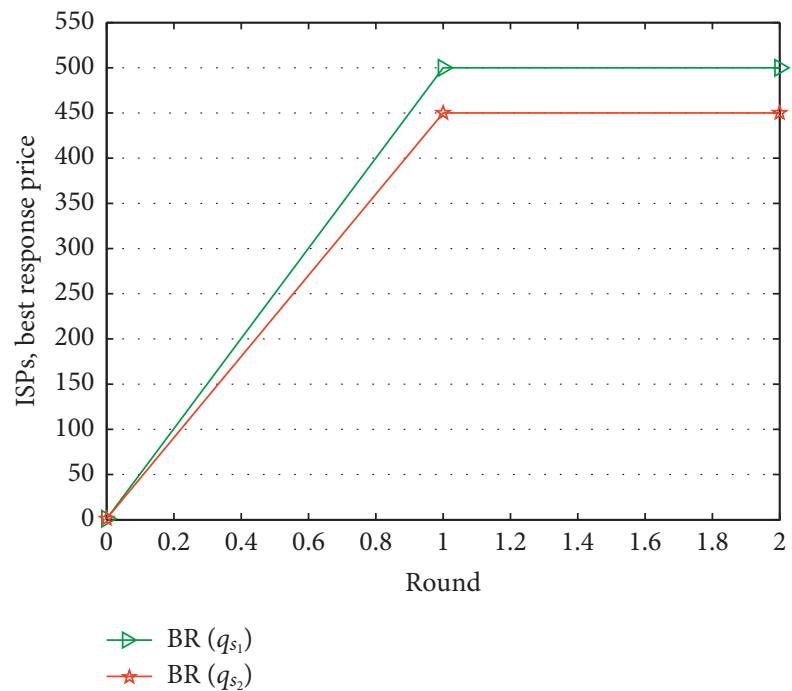

FIGURE 5: Nash equilibrium QoS under the best response dynamic.

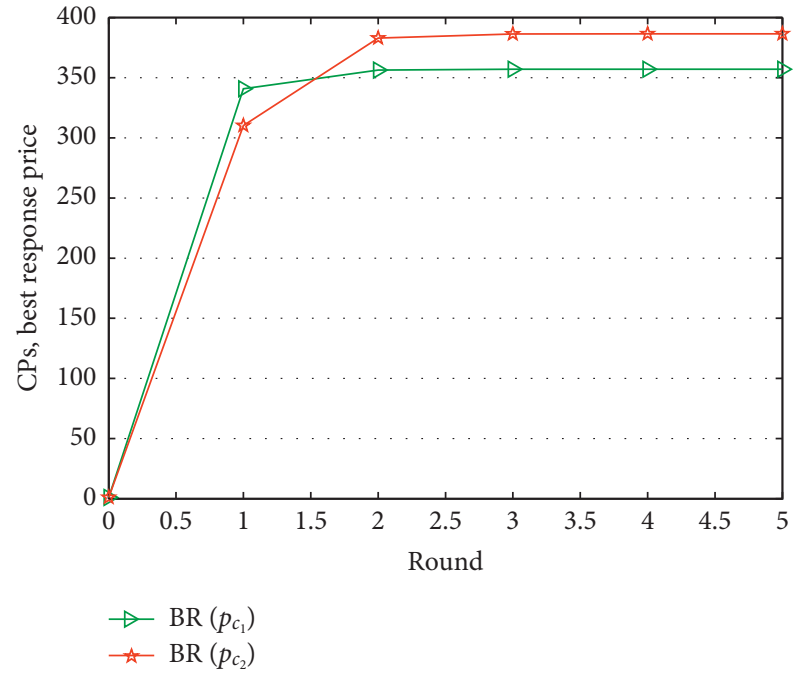

FIGURE 6: Nash equilibrium price under the best response dynamic.

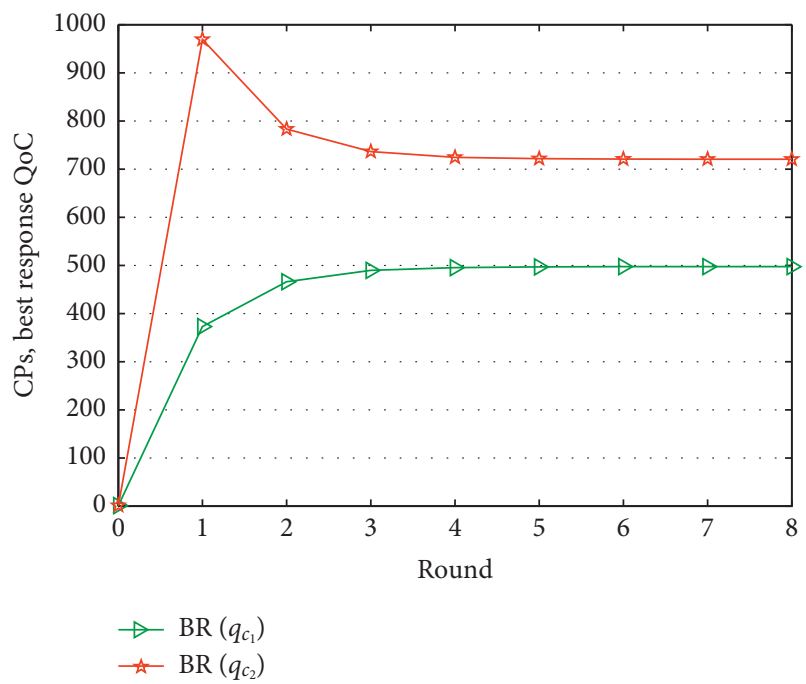

Figure 7: Nash equilibrium QoC under the best response dynamic.

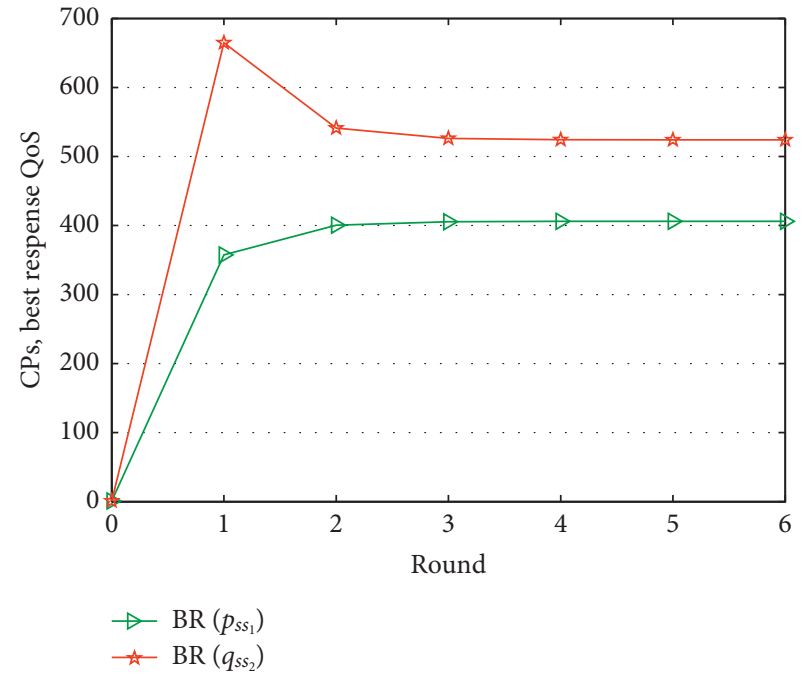

FIgURE 8: Nash equilibrium QoS under the best response dynamic. 


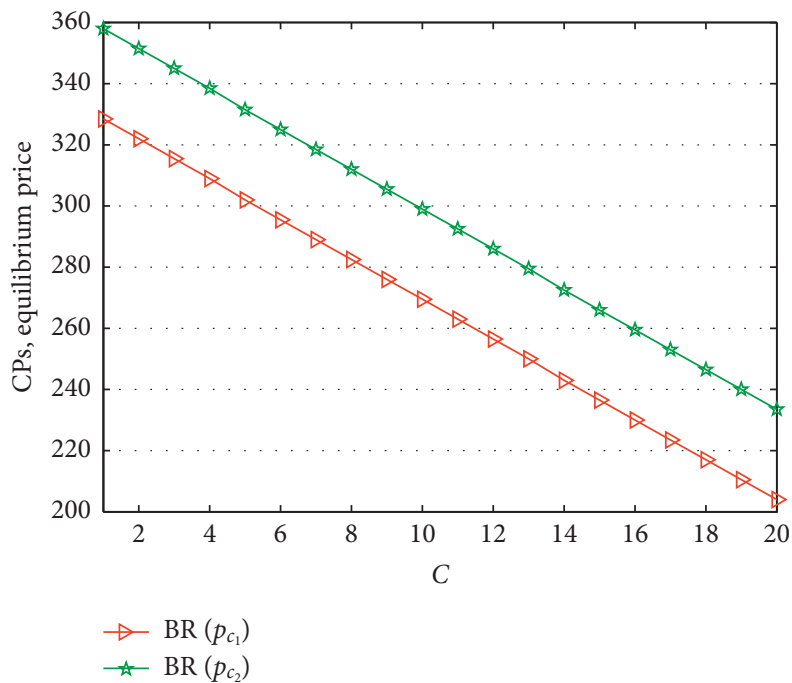

Figure 9: Nash equilibrium price $p_{c}$ evolution with respect to caching cost $C$.

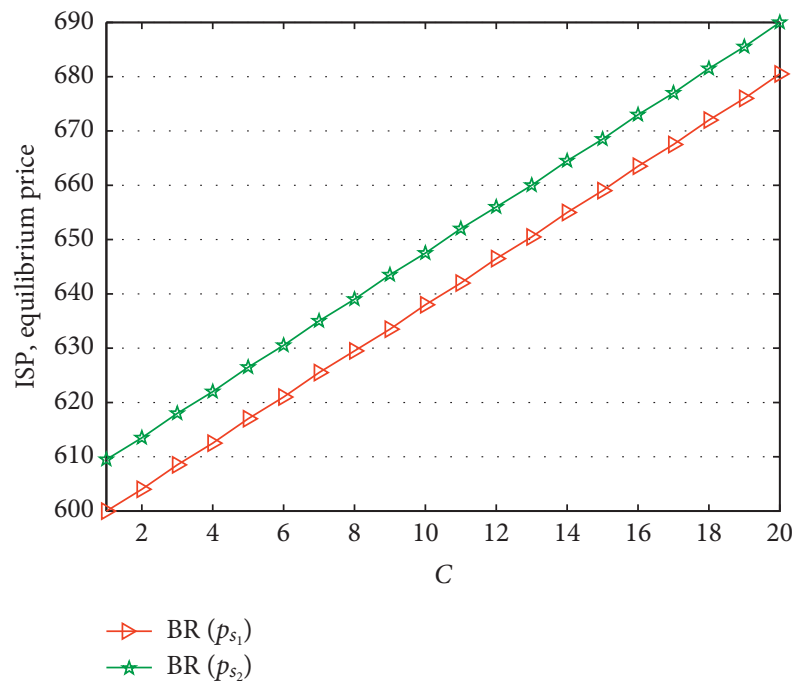

Figure 10: Nash equilibrium price $p_{s}$ evolution with respect to caching cost $C$.

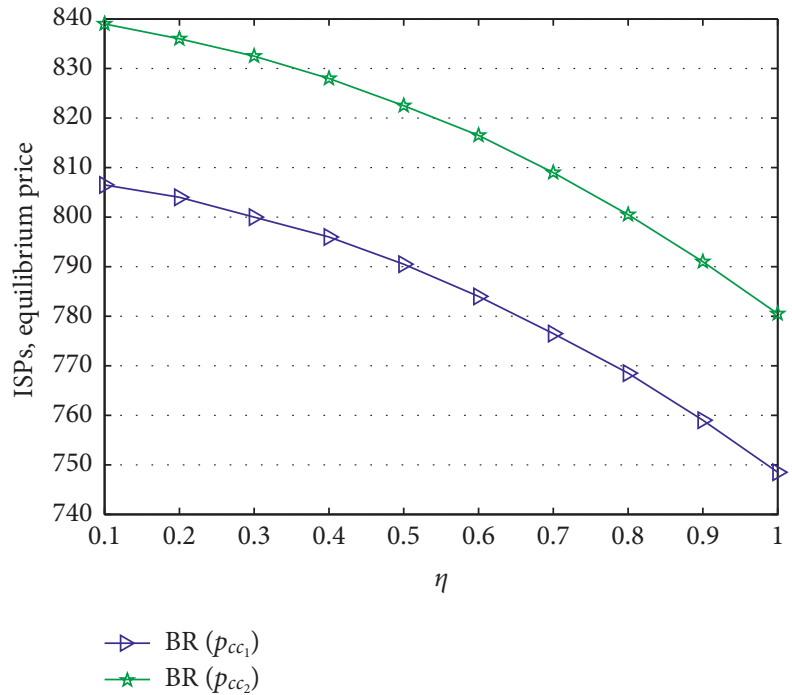

FIGURE 11: Nash equilibrium price $p_{c c}$ evolution as a function of Zipf's factor $\eta$. 


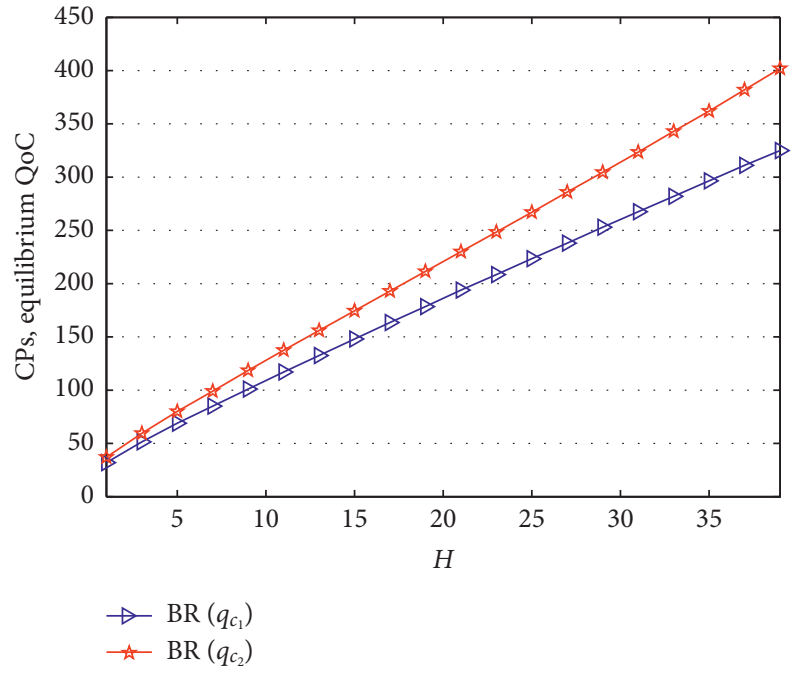

FIgURE 12: Nash equilibrium QoC $q_{c}$ evolution with respect to $H$.

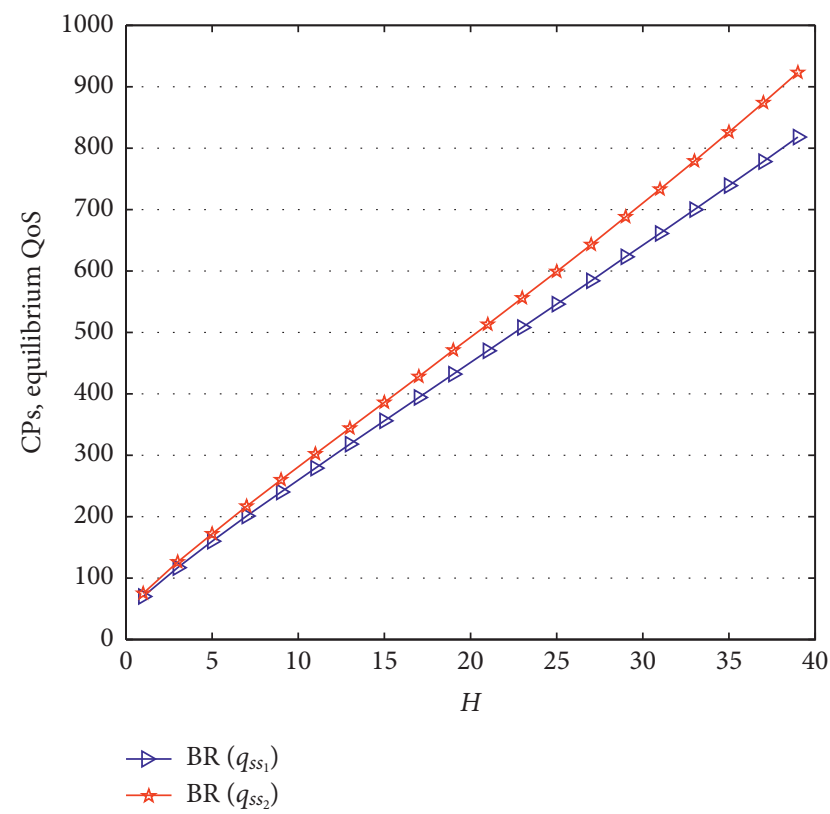

FIgURE 13: Nash equilibrium QoS $q_{c}$ evolution as a function of $H$.

respect to $C$. When $C$ is lower, the PoA is lower; then, the Nash equilibrium is not socially efficient, the ISPs are selfish, and each one seeks to maximize its profit individually. However, when $C$ increases, the equilibrium becomes more and more socially efficient; this increase finds the simple intuition that when $C$ increases, ISPs cooperate with each other for optimizing Nash equilibrium.

Figure 16 shows the ISP PoA variation curve as a function of the number of items cached $H$. The price of anarchy decreases with respect to $H$. When $H$ is lower, the price of anarchy is socially efficient; moreover, when $H$ is lower, the income from caching decreases and ISPs cooperate for optimizing the Nash equilibrium. On the other hand, when $H$ increases, the price of anarchy is lower; then,

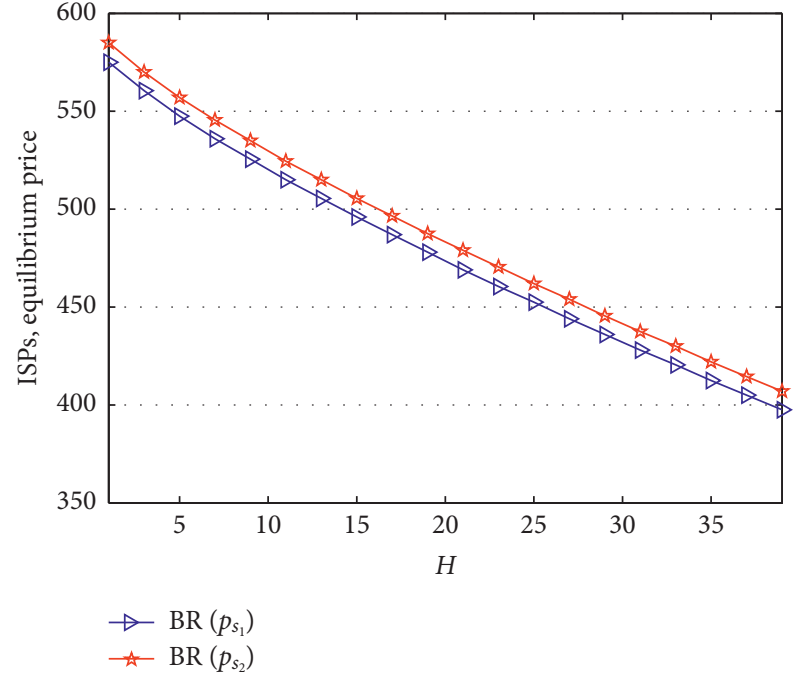

FIgURE 14: Nash equilibrium price $p_{s}$ evolution as a function of $H$.

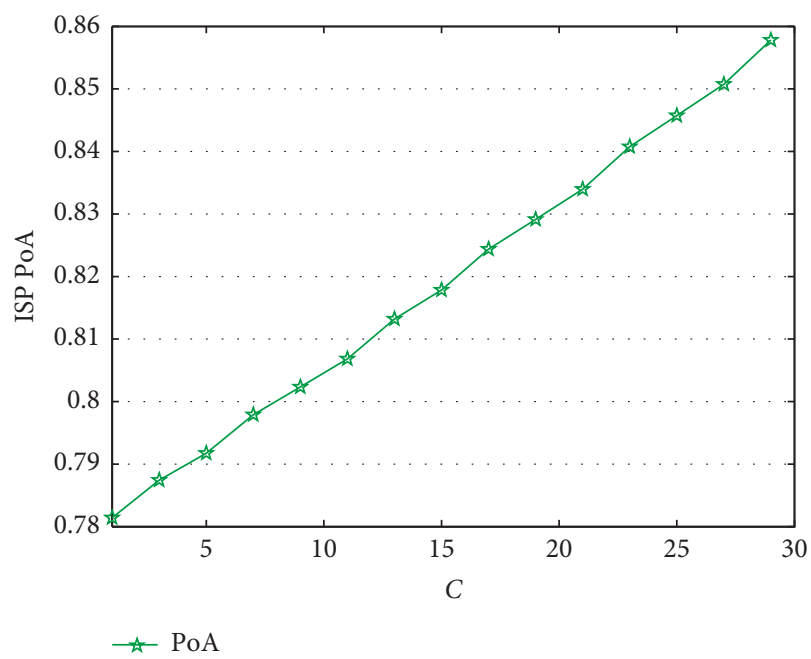

FIGURE 15: Price of anarchy evolution with respect to caching cost C.

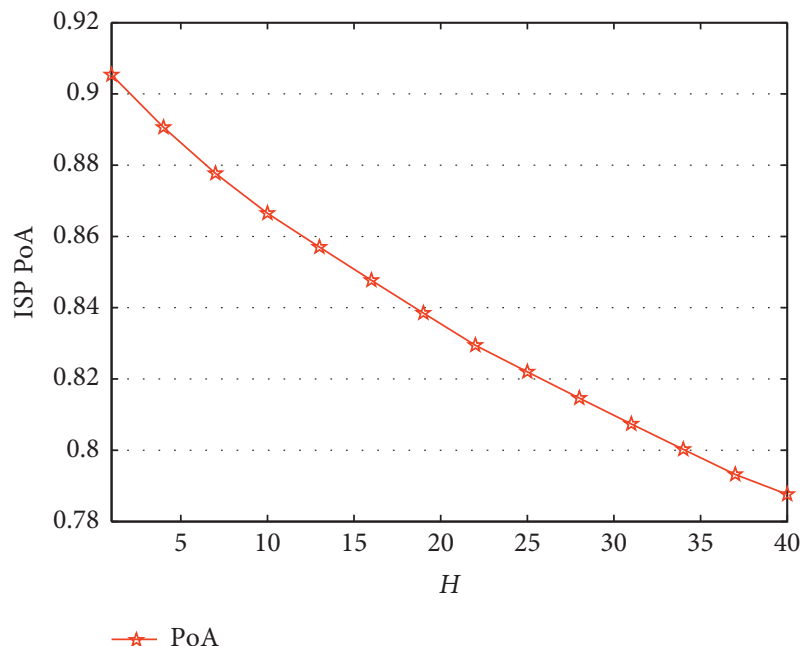

FIgURe 16: Price of anarchy evolution with respect to $H$. 


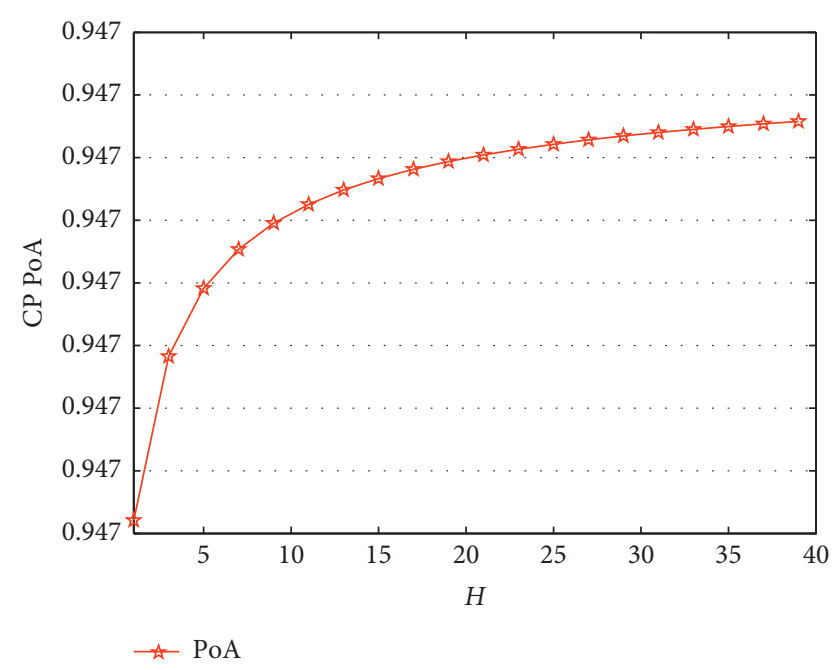

Figure 17: Price of anarchy evolution with respect to $H$.

the Nash equilibrium is not socially efficient, ISPs are selfish, and each one seeks to maximize its profit individually.

Figure 17 shows the CP PoA variation curve as a function of $H$. The price of anarchy increases with respect to $H$. A special feature is that the Nash equilibrium performs well, and the loss of efficiency is only around 6\%. This result indicates that the Nash equilibrium of this game is fair and socially efficient.

\section{Conclusion}

In this paper, we developed an analytical framework for the distribution of accessible content in an ICN that comprises multiple ISPs and multiple CPs. The interaction among ISPs and among CPs is investigated by using the noncooperative game. Additionally, we have used a Stackelberg game to study the interaction between CP and ISP. Each ISP can control the amount of content cached in the network and adjust its strategies (network access price, price to access to content in the cache, QoS, and credibility of content in the cache). The CP can adjust its strategy (content access price and credibility of content). We consider that the popularity of content follows a generalized Zipf distribution. We proved the existence and uniqueness of the Nash equilibrium in a competitive ICN market. This result is significant because it implies that a stable solution with suitable economic incentives in collaborative caching is feasible in the ICN paradigm. Next, we describe a learning mechanism that allows each ISP to discover accurately and rapidly its equilibrium policies. Also, we use the price of anarchy to evaluate the effectiveness of the Nash equilibrium. The simulation results show that caching investment is beneficial for the ISPs, the CPs, and end-users.

\section{Appendix}

\section{A. Proof of Theorem 2}

The sufficient condition of existing at least one equilibrium, if and only if its second derivative of the utility $U_{\mathrm{ISP}_{j}}$ function with respect to price $p_{c c_{j}}$ is nonpositive. Indeed, we first compute the first derivative of $U_{\mathrm{ISP}_{j}}$ :

$$
\begin{aligned}
\frac{\partial U_{\mathrm{ISP}_{j}}}{\partial p_{c c_{j}}}= & -N \rho_{j}^{j} p_{s_{j}}+\sum_{i=1}^{N} \sum_{h=1}^{H} \phi_{h}\left\{-\rho_{j}^{j} \tau_{j}^{i} k_{i j h}\left(p_{c c_{j}}-C_{i h}\right)+\tau_{j}^{i} k_{i j h} D_{i j}\right. \\
& \left.+\rho_{j}^{j} p_{t_{i}} c_{i}\left(1-\tau_{j}^{i} k_{i j h}\right)\right\}+v_{j} N \rho_{j}^{j}\left(H N-\sum_{i=1}^{N} \tau_{j}^{i} \sum_{h=1}^{H} k_{i j h}\right) .
\end{aligned}
$$

Then, we calculate the second derivative:

$$
\frac{\partial^{2} U_{\text {ISP }_{j}}}{\partial p_{c c_{j}}^{2}}=-2 \rho_{j}^{j} \sum_{i=1}^{N} \sum_{h=1}^{H} \phi_{h} \tau_{j}^{i} k_{i j h} \leq 0,
$$

which ensures existence of a Nash equilibrium.

The mixed partial is written as

$$
\frac{\partial^{2} U_{\mathrm{ISP}_{j}}}{\partial p_{c c_{j}} \partial p_{c c_{f}}}=\rho_{j}^{f} \sum_{i=1}^{N} \sum_{h=1}^{H} \phi_{h} \tau_{j}^{i} k_{i j h} .
$$

Then,

$$
\begin{aligned}
& -\frac{\partial^{2} U_{\mathrm{ISP}_{j}}}{\partial p_{c c_{j}}^{2}}-\sum_{f=1, f \neq j}^{F}\left|\frac{\partial^{2} U_{\mathrm{ISP}_{j}}}{\partial p_{c c_{j}} \partial p_{c c_{f}}}\right|=\left(2 \rho_{j}^{j}-\sum_{f=1, f \neq j}^{F} \rho_{j}^{f}\right) \\
& \quad \cdot \sum_{i=1}^{N} \sum_{h=1}^{M} \phi_{h} \tau_{j}^{i} k_{i j h} \geq 0 .
\end{aligned}
$$

Thus, the game $G_{1}\left(\mathbf{p}_{s}, \mathbf{q}_{s}\right)$ admits a unique Nash equilibrium point.

\section{B. Proof of Theorem 3}

The sufficient condition of existing at least one equilibrium, if and only if its second derivative of the utility $U_{\text {ISP }_{j}}$ function with respect to QoS $q_{s_{j}}$ is nonpositive. Indeed, we first compute the first derivative of $U_{\text {ISP }}$ :

$$
\begin{aligned}
\frac{\partial U_{\mathrm{ISP}_{j}}}{\partial q_{s_{j}}}= & N \beta_{j}^{j} p_{s_{j}}+\sum_{i=1}^{N} \sum_{h=1}^{H} \phi_{h}\left\{\beta_{j}^{j} \tau_{j}^{i} k_{i j h}\left(p_{c c_{j}}-C_{i h}\right)\right. \\
& \left.-\beta_{j}^{j} p_{t_{i}} c_{i}\left(1-\tau_{j}^{i} k_{i j h}\right)\right\} \\
& -v_{j}\left(2 q_{s_{j}}+N \beta_{j}^{j}\right)\left(N H-\sum_{i=1}^{N} \tau_{j}^{i} \sum_{h=1}^{H} k_{i j h}\right) .
\end{aligned}
$$

Then,

$$
\frac{\partial^{2} U_{\mathrm{ISP}_{j}}}{\partial q_{s_{j}}^{2}}=-2 v_{j}\left(N H-\sum_{i=1}^{N} \tau_{j}^{i} \sum_{h=1}^{H} k_{i j h}\right) \leq 0,
$$

which ensures existence of a Nash equilibrium.

In order to prove uniqueness, we follow [40] and define the weighted sum of user utility functions: 


$$
\psi\left(\mathbf{q}_{s}, \mathbf{x}\right)=\sum_{j=1}^{F} x_{j} U_{\operatorname{ISP}_{j}}\left(q_{s_{j}}, \mathbf{q}_{s_{-j}}\right) .
$$

The Jacobian matrix $J$ of the pseudogradient $v\left(\mathbf{q}_{s}, \mathbf{x}\right)$ is written as

The pseudogradient of (B.3) is given by

$$
v\left(\mathbf{q}_{s}, \mathbf{x}\right)=\left[x_{1} \nabla U_{1}\left(q_{s_{1}}, \mathbf{q}_{s_{-1}}\right), \ldots, x_{F} \nabla U_{F}\left(q_{s_{F}}, \mathbf{q}_{s_{-F}}\right)\right]^{T} .
$$

$$
J=\left(\begin{array}{cccc}
x_{1} \frac{\partial^{2} U_{\mathrm{ISP}}}{\partial q_{s_{1}}^{2}} & x_{1} \frac{\partial^{2} U_{\mathrm{ISP}_{1}}}{\partial q_{s_{1}} \partial q_{s_{2}}} & \cdots & x_{1} \frac{\partial^{2} U_{\mathrm{ISP}}}{\partial q_{s_{1}} \partial q_{s_{F}}} \\
x_{2} \frac{\partial^{2} U_{\mathrm{ISP}_{2}}}{\partial q_{s_{2}} \partial q_{s_{1}}} & x_{2} \frac{\partial^{2} U_{\mathrm{ISP}}}{\partial p_{s_{2}}^{2}} & \cdots & x_{2} \frac{\partial^{2} U_{\mathrm{ISP}}}{\partial q_{s_{2}} \partial q_{s_{F}}} \\
\cdot & \cdot & \cdot \\
\cdot & \cdot & \cdot \\
\cdot & \cdot & \cdot \\
x_{M} \frac{\partial^{2} U_{\mathrm{ISP}_{F}}}{\partial q_{s_{F}} \partial q_{s_{1}}} & x_{F} \frac{\partial^{2} U_{\mathrm{ISP}_{F}}}{\partial q_{s_{F}} \partial q_{s_{2}}} & \cdot & x_{F} \frac{\partial^{2} U_{\mathrm{ISP}}}{\partial q_{s_{F}}^{2}}
\end{array}\right)
$$

$$
\begin{aligned}
& \left(-2 x_{1} v_{1} \sum_{i=1}^{N}\left(1-\tau_{1}^{i} \sum_{h=1}^{H} k_{i 1 h}\right)\right. \\
& \text { Thus, } J \text { is a diagonal matrix with negative diagonal el- } \\
& \text { ements. This implies that } J \text { is negative definite. Henceforth, } \\
& {\left[J+x_{2} v_{2} \sum_{i=1}^{N}\left(1-\tau_{2}^{i}\right. \text { is also negative definite, and according to Theorem }\right.} \\
& \text { (6) in, [40], the weighted sum of the utility functions } \psi\left(\mathbf{q}_{s}, \mathbf{x}\right) \\
& \text { is diagonally strictly concave. Thus, the Nash equilibrium }
\end{aligned}
$$
point is unique.

\section{Proof of Theorem 4}

The sufficient condition of existing at least one equilibrium, if and only if its second derivative of the utility $U_{\mathrm{CP}_{i}}$ function with respect to price $p_{c_{i}}$ is nonpositive. Indeed, we first compute the first derivative of $U_{\mathrm{CP}_{i}}$ :

$$
\begin{aligned}
\frac{\partial U_{\mathrm{CP}_{i}}}{\partial p_{c_{i}}}= & \sum_{j=1}^{F} \sum_{h=1}^{H} \phi_{h}\left\{D_{i j}\left(1-\tau_{j}^{i} k_{i j h}\right)-\delta_{i}^{i}\left(1-\tau_{j}^{i} k_{i j h}\right)\right. \\
& \left.\cdot\left(p_{c_{i}}+p_{t_{i}} c_{i}\right)-\delta_{i}^{i} \tau_{j}^{i} k_{i j h} C_{i h}\right\} .
\end{aligned}
$$

Then, we calculate the second derivative of $U_{\mathrm{CP}_{i}}$ :

$$
\frac{\partial^{2} U_{\mathrm{CP}_{i}}}{\partial p_{c_{i}}^{2}}=-2 \delta_{i}^{i} \sum_{j=1}^{F} \sum_{h=1}^{H} \phi_{h}\left(1-\tau_{j}^{i} k_{i j h}\right) \leq 0,
$$

which ensures existence of a Nash equilibrium.

The mixed partial is written as 


$$
\frac{\partial^{2} U_{\mathrm{CP}_{i}}}{\partial p_{c_{i}} \partial p_{c_{n}}}=\delta_{i}^{n} \sum_{j=1}^{F} \sum_{h=1}^{H} \phi_{h}\left(1-\tau_{j}^{i} k_{i j h}\right)
$$

Then,

$$
\begin{gathered}
-\frac{\partial^{2} U_{\mathrm{CP}_{i}}}{\partial p_{c_{i}}^{2}}-\sum_{n=1, n \neq i}^{N}\left|\frac{\partial^{2} U_{\mathrm{CP}_{i}}}{\partial p_{c_{i}} \partial p_{c_{n}}}\right|=\left(\delta_{i}^{i}-\sum_{n=1, n \neq i}^{N} \delta_{i}^{n}\right) \\
\cdot \sum_{j=1}^{F} \sum_{h=1}^{H} \phi_{h}\left(1-\tau_{j}^{i} k_{i j h}\right) \geq 0 .
\end{gathered}
$$

Thus, the game $G_{2}\left(\mathbf{q}_{s s}, \mathbf{q}_{c}\right)$ admits a unique Nash equilibrium point.

\section{Proof of Theorem 5}

The sufficient condition of existing at least one equilibrium, if and only if its second derivative of the utility $U_{\mathrm{CP}}$ function with respect to QoC $q_{c_{i}}$ is nonpositive. Indeed, we first compute the first derivative of $U_{\mathrm{CP}_{i}}$ :

$$
\begin{aligned}
\frac{\partial U_{\mathrm{CP}_{i}}}{\partial q_{c_{i}}}= & \sum_{j=1}^{F} \sum_{h=1}^{H} \phi_{h}\left\{\mu \sigma_{i}^{i} p_{c_{i}}-\frac{\mu \sigma_{i}^{i} p_{c_{i}} k_{i j h} c_{j}}{\lambda q_{s s_{i}}+\mu q_{c_{i}}}+\frac{\mu p_{c_{i}} D_{i j} k_{i j h} c_{j}}{\left(\lambda q_{s s_{i}}+\mu q_{c_{i}}\right)^{2}}+\mu p_{t_{i}} D_{i j}\right. \\
& \left.+\mu p_{t_{i}} \sigma_{i}^{i}\left(\lambda q_{s s_{i}}+\mu q_{c_{i}}\right)-\mu \sigma_{i}^{i} c_{j} p_{t_{i}} k_{i j h}+\frac{\mu k_{i j h} C_{i h} \sigma_{i}^{i} c_{j}}{\lambda q_{s s_{i}}+\mu q_{c_{i}}}-\frac{\mu k_{i j h} C_{i h} D_{i j} c_{j}}{\left(\lambda q_{s s_{i}}+\mu q_{c_{i}}\right)^{2}}\right\} \\
& -2 \mu \theta_{i}\left(\lambda q_{s s_{i}}+\mu q_{c_{i}}\right) .
\end{aligned}
$$

Then,

$$
\frac{\partial^{2} U_{\mathrm{CP}_{i}}}{\partial q_{c_{i}}^{2}}=\sum_{j=1}^{F} \sum_{h=1}^{H} \phi_{h}\left\{2 \mu^{2} \sigma_{i}^{i} p_{t_{i}}+\frac{2 \mu^{2} k_{i j h} c_{j}}{\left(\lambda q_{s s_{i}}+\mu q_{c_{i}}\right)^{2}}\left(\frac{D_{i j}}{\lambda q_{s s_{i}}+\mu q_{c_{i}}}-\sigma_{i}^{i}\right)\left(C_{i h}-p_{c_{i}}\right)\right\}-2 \mu^{2} \theta_{i} .
$$

If $\quad p_{c_{i}} \geq C_{i h} \quad$ and $\quad\left(2 \mu^{2} k_{i j h} c_{j} /\left(\lambda q_{s s_{i}}+\mu q_{c_{i}}\right)^{2}\right)$ $\left(\left(D_{i j} /\left(\lambda q_{s s_{i}}+\mu q_{c_{i}}\right)\right)-\sigma_{i}^{i}\right)\left(C_{i h}-p_{c_{i}}\right) \geq 2 \sigma_{i}^{i} p_{t_{i}}$, then

$$
\frac{\partial^{2} U_{\mathrm{CP}_{i}}}{\partial q_{c_{i}}^{2}} \leq 0
$$

The second derivative of the utility function is always nonpositive, then the utility function is concave, which ensures the existence of a Nash equilibrium.

The mixed partial is written as

$$
\frac{\partial^{2} U_{\mathrm{CP}_{i}}}{\partial q_{c_{i}} \partial q_{c_{n}}}=\sum_{j=1}^{F} \sum_{h=1}^{H} \phi_{h}\left\{-\mu^{2} \sigma_{i}^{n} p_{t_{i}}+\frac{\sigma_{i}^{n} \mu^{2} k_{i j h} c_{j}}{\left(\lambda q_{s s_{i}}+\mu q_{c_{i}}\right)^{2}}\left(C_{i h}-p_{c_{i}}\right)\right\} \text {. }
$$
then

We have $\sigma_{i}^{n} p_{t_{i}} \geq\left(\mu^{2} k_{i j h} \sigma_{i}^{n} c_{j} /\left(\lambda q_{s s_{i}}+\mu q_{c_{i}}\right)^{2}\right)\left(C_{i h}-p_{c_{i}}\right)$,

$$
\left|\frac{\partial^{2} U_{\mathrm{CP}_{i}}}{\partial q_{c_{i}} \partial q_{c_{n}}}\right|=\sum_{j=1}^{F} \sum_{h=1}^{H} \phi_{h}\left\{\mu^{2} \sigma_{i}^{n} p_{t_{i}}-\frac{\mu^{2} k_{i j h} \sigma_{i}^{n} c_{j}}{\left(\lambda q_{s s_{i}}+\mu q_{c_{i}}\right)^{2}}\left(C_{i h}-p_{c_{i}}\right)\right\} .
$$

Thus,

$$
\begin{gathered}
-\frac{\partial^{2} U_{\mathrm{CP}_{i}}}{\partial q_{c_{i}}^{2}}-\sum_{n=1, n \neq i}^{N}\left|\frac{\partial^{2} U_{\mathrm{CP}_{i}}}{\partial q_{c_{i}} \partial q_{c_{n}}}\right|=\sum_{j=1}^{F} \sum_{h=1}^{H} \phi_{h} \\
\cdot\left\{-\mu^{2} p_{t_{i}}-\frac{k_{i j h} \mu^{2} \sigma_{i}^{n} c_{j}}{\left(\lambda q_{s s_{i}}+\mu q_{c_{i}}\right)^{2}}\left(C_{i h}-p_{c_{i}}\right)\right. \\
\left.\quad \times\left(2 \frac{D_{i j}}{\lambda q_{s s_{i}}+\mu q_{c_{i}}}-2 \sigma_{i}^{i}+\sum_{n=1, n \neq i}^{N} \sigma_{i}^{n}\right)\right\} .
\end{gathered}
$$

We have $-\left(k_{i j h} \sigma_{i}^{n} c_{j} /\left(\lambda q_{s s_{i}}+\mu q_{c_{i}}\right)^{2}\right)\left(C_{i h}-p_{c_{i}}\right)\left(2\left(D_{i j} /\right.\right.$ $\left.\left.\left(\lambda q_{s s_{i}}+\mu q_{c_{i}}\right)\right)-2 \sigma_{i}^{i}+\sum_{n=1, n \neq i}^{N} \sigma_{i}^{n}\right) \geq p_{t_{i}}$, and then

$$
-\frac{\partial^{2} U_{\mathrm{CP}_{i}}}{\partial q_{c_{i}}^{2}}-\sum_{n=1, n \neq i}^{N}\left|\frac{\partial^{2} U_{\mathrm{CP}_{i}}}{\partial q_{c_{i}} \partial q_{c_{n}}}\right| \geq 0 .
$$

Thus, the game $G_{2}\left(\mathbf{p}_{c}, \mathbf{q}_{s s}\right)$ admits a unique Nash equilibrium point.

\section{E. Proof of Theorem 6}

The sufficient condition of existing at least one equilibrium, if and only if its second derivative of the utility $U_{\mathrm{CP}_{i}}$ function 
with respect to $\mathrm{QoC} q_{s s_{i}}$ is nonpositive. Indeed, we first compute the first derivative of $U_{\mathrm{CP}_{i}}$ :

$$
\begin{aligned}
\frac{\partial U_{\mathrm{CP}_{i}}}{\partial q_{s s_{i}}}= & \sum_{j=1}^{F} \sum_{h=1}^{H} \phi_{h}\left\{\lambda \sigma_{i}^{i} p_{c_{i}}-\frac{\lambda \sigma_{i}^{i} p_{c_{i}} k_{i j h} c_{j}}{\lambda q_{s s_{i}}+\mu q_{c_{i}}}+\frac{\lambda p_{c_{i}} D_{i j} k_{i j h} c_{j}}{\left(\lambda q_{s s_{i}}+\mu q_{c_{i}}\right)^{2}}+\lambda p_{t_{i}} D_{i j}+\lambda p_{t_{i}} \sigma_{i}^{i}\left(\lambda q_{s s_{i}}+\mu q_{c_{i}}\right)\right. \\
& \left.-\lambda \sigma_{i}^{i} c_{j} p_{t_{i}} k_{i j h}+\frac{\lambda k_{i j h} C_{i h} \sigma_{i}^{i} c_{j}}{\lambda q_{s s_{i}}+\mu q_{c_{i}}}-\frac{\lambda k_{i j h} C_{i h} D_{i j} c_{j}}{\left(\lambda q_{s s_{i}}+\lambda q_{c_{i}}\right)^{2}}\right\}-2 \lambda \theta_{i}\left(\lambda q_{s s_{i}}+\mu q_{c_{i}}\right) .
\end{aligned}
$$

Then,

$$
\frac{\partial^{2} U_{\mathrm{CP}_{i}}}{\partial q_{s s_{i}}^{2}}=\sum_{j=1}^{F} \sum_{h=1}^{H} \phi_{h}\left\{2 \lambda^{2} \sigma_{i}^{i} p_{t_{i}}+\frac{2 \lambda^{2} k_{i j h} c_{j}}{\left(\lambda q_{s s_{i}}+\mu q_{c_{i}}\right)^{2}}\left(\frac{D_{i j}}{\lambda q_{s s_{i}}+\mu q_{c_{i}}}-\sigma_{i}^{i}\right)\left(C_{i h}-p_{c_{i}}\right)\right\}-2 \lambda^{2} \theta_{i} .
$$

If $\quad p_{c_{i}} \geq C_{i h}$ and $\left(2 \lambda^{2} k_{i j h} c_{j} /\left(\lambda q_{s s_{i}}+\mu q_{c_{i}}\right)^{2}\right)\left(\left(D_{i j} /\right.\right.$ $\left.\left.\left(\lambda q_{s s_{i}}+\mu q_{c_{i}}\right)\right)-\sigma_{i}^{i}\right)\left(C_{i h}-p_{c_{i}}\right) \geq 2 \sigma_{i}^{i} p_{t_{i}}$, then

$$
\frac{\partial^{2} U_{\mathrm{CP}_{i}}}{\partial q_{s s_{i}}^{2}} \leq 0
$$

which ensures existence of a Nash equilibrium.

The mixed partial is written as

$$
\frac{\partial^{2} U_{\mathrm{CP}_{i}}}{\partial q_{s s_{i}} \partial q_{s s_{n}}}=\sum_{j=1}^{F} \sum_{h=1}^{H} \phi_{h}\left\{-\lambda^{2} \sigma_{i}^{n} p_{t_{i}}+\frac{\sigma_{i}^{n} \lambda^{2} k_{i j h} c_{j}}{\left(\lambda q_{s s_{i}}+\mu q_{c_{i}}\right)^{2}}\left(C_{i h}-p_{c_{i}}\right)\right\} \text {. }
$$

We have $\sigma_{i}^{n} p_{t_{i}} \geq\left(\lambda^{2} k_{i j h} \sigma_{i}^{n} c_{j} /\left(\lambda q_{s s_{i}}+\mu q_{c_{i}}\right)^{2}\right)\left(C_{i h}-p_{c_{i}}\right)$, and then

$$
\left|\frac{\partial^{2} U_{C P_{i}}}{\partial q_{s s_{i}} \partial q_{s s_{n}}}\right|=\sum_{j=1}^{F} \sum_{h=1}^{H} \phi_{h}\left\{\lambda^{2} \sigma_{i}^{n} p_{t_{i}}-\frac{\lambda^{2} k_{i j h} \sigma_{i}^{n} c_{j}}{\left(\lambda q_{s s_{i}}+\mu q_{c_{i}}\right)^{2}}\left(C_{i h}-p_{c_{i}}\right)\right\} .
$$

Thus,

$$
-\frac{\partial^{2} U_{\mathrm{CP}_{i}}}{\partial q_{s s_{i}}^{2}}-\sum_{n=1, n \neq i}^{N}\left|\frac{\partial^{2} U_{\mathrm{CP}_{i}}}{\partial q_{s s_{i}} \partial q_{s s_{n}}}\right|=\sum_{j=1}^{F} \sum_{h=1}^{H} \phi_{h}\left\{-\lambda^{2} p_{t_{i}}-\frac{k_{i j h} \lambda^{2} \sigma_{i}^{n} c_{j}}{\left(\lambda q_{s s_{i}}+\lambda q_{c_{i}}\right)^{2}}\left(C_{i h}-p_{c_{i}}\right) \times\left(2 \frac{D_{i j}}{\lambda q_{s s_{i}}+\lambda q_{c_{i}}}-2 \sigma_{i}^{i}+\sum_{n=1, n \neq i}^{N} \sigma_{i}^{n}\right)\right\} .
$$

We have $-\left(k_{i j h} \sigma_{i}^{n} c_{j} /\left(\lambda q_{s s_{i}}+\mu q_{c_{i}}\right)^{2}\right)\left(C_{i h}-p_{c_{i}}\right)\left(2\left(D_{i j} /\right.\right.$ $\left.\left.\lambda q_{s s_{i}}+\mu q_{c_{i}}\right)-2 \sigma_{i}^{i}+\sum_{n=1, n \neq i}^{N} \sigma_{i}^{n}\right) \geq p_{t_{i}}$, and then

$$
-\frac{\partial^{2} U_{\mathrm{CP}_{i}}}{\partial q_{s s_{i}}^{2}}-\sum_{n=1, n \neq i}^{N}\left|\frac{\partial^{2} U_{\mathrm{CP}_{i}}}{\partial q_{s s_{i}} \partial q_{s s_{n}}}\right| \geq 0 .
$$

Thus, the game $G_{2}\left(\mathbf{p}_{c}, \mathbf{q}_{c}\right)$ admits a unique Nash equilibrium point.

\section{Data Availability}

The data used to support the findings of this study are available from the corresponding author upon request.

\section{Conflicts of Interest}

The authors declare that there are no conflicts of interest regarding the publication of this paper.

\section{References}

[1] B. Ahlgren, C. Dannewitz, C. Imbrenda, D. Kutscher, and B. Ohlman, "A survey of information-centric networking," IEEE Communications Magazine, vol. 50, p. 7, 2012.

[2] A. Gharaibeh, A. Khreishah, I. Khalil, and J. Wu, "Asymptotically-optimal incentive-based en-route caching scheme," in Proceedings of the 2014 IEEE 11th International Conference 
on Mobile Ad Hoc and Sensor Systems, pp. 318-326, IEEE, Philadelphia, PA, USA, October 2014.

[3] T.-M. Pham, S. Fdida, and P. Antoniadis, "Pricing in information-centric network interconnection," in Proceedings of the IFIP Networking Conference, pp. 1-9, IEEE, Brooklyn, NY, USA, May 2013.

[4] N. Zhang, T. Leva, and H. Hammainen, "Two-sidedness of internet content delivery," in Proceedings of the 10th Conference on Telecommunication, Media and Internet TechnoEconomics (CTTE), pp. 1-6, VDE, Berlin, Germany, May 2011.

[5] H. Garmani, M. Outanoute, M. Baslam, and M. Jourhmane, "New competition-based approach for caching popular content in ICN," in Networked Systems, A. Podelski and F. Taïani, Eds., vol. 11028, pp. 286-300, Springer International Publishing, Cham, Switzerland, 2019.

[6] K. Hamidouche, W. Saad, M. Debbah, and H. V. Poor, "Mean-field games for distributed caching in ultra-dense small cell networks," in Proceedings of the 2016 American Control Conference (ACC), pp. 4699-4704, IEEE, Boston, MA, USA, July 2016.

[7] T. D. Tran and L. B. Le, "Joint wireless access-backhaul network slicing and content caching optimization," in Proceedings of the 2018 IEEE International Conference on Communications Workshops (ICC Workshops), pp. 1-6, IEEE, Kansas City, MO, USA, May 2018.

[8] Z. Zhang, C.-H. Lung, I. Lambadaris, and M. St-Hilaire, "When 5G meets ICN: an ICN-based caching approach for mobile video in $5 \mathrm{G}$ networks," Computer Communications, vol. 118, pp. 81-92, 2018.

[9] G. Quer, I. Pappalardo, B. D. Rao, and M. Zorzi, "Proactive caching strategies in heterogeneous networks with device-todevice communications," IEEE Transactions on Wireless Communications, vol. 17, no. 8, pp. 5270-5281, 2018.

[10] Y. Zhang, Y. Xu, Q. Wu, X. Liu, K. Yao, and A. Anpalagan, “A game-theoretic approach for optimal distributed cooperative hybrid caching in D2d networks," IEEE Wireless Communications Letters, vol. 7, no. 3, pp. 324-327, 2018.

[11] B. Assila, A. Kobbane, M. Elmachkour, and M. E. Koutbi, "A dynamic stackelberg-cournot game for competitive content caching in 5G networks," in Proceedings of the 2017 International Conference on Wireless Networks and Mobile Communications (WINCOM), pp. 1-6, IEEE, Rabat, Morocco, November 2017.

[12] Z. Su, Y. Hui, Q. Xu, T. Yang, J. Liu, and Y. Jia, "An edge caching scheme to distribute content in vehicular networks," IEEE Transactions on Vehicular Technology, vol. 67, no. 6, pp. 5346-5356, 2018.

[13] Y. Lu, W. Chen, and H. V. Poor, "Coded joint pushing and caching with asynchronous user requests," IEEE Journal on Selected Areas in Communications, vol. 36, no. 8, pp. 18431856, 2018.

[14] K. Zhang, S. Leng, Y. He, S. Maharjan, and Y. Zhang, "Cooperative content caching in $5 \mathrm{G}$ networks with mobile edge computing," IEEE Wireless Communications, vol. 25, no. 3, pp. 80-87, 2018.

[15] X. Shao, H. Asaeda, M. Dong, and Z. Ma, "Cooperative interdomain cache sharing for information-centric networking via a bargaining game approach," IEEE Transactions on Network Science and Engineering, vol. 6, no. 4, pp. 698-710, 2019.

[16] M. Hajimirsadeghi, N. B. Mandayam, and A. Reznik, "Joint caching and pricing strategies for popular content in information centric networks," IEEE Journal on Selected Areas in Communications, vol. 35, no. 3, pp. 654-667, 2017.
[17] J. Künsemöller, N. Zhang, K. Berg, and J. Soares, "A gametheoretic evaluation of an ISP business model in caching," Information Systems Frontiers, vol. 19, no. 4, pp. 803-818, 2016.

[18] A. Ndikumana, N. H. Tran, T. M. Ho, D. Niyato, Z. Han, and C. S. Hong, "Joint incentive mechanism for paid content caching and price based cache replacement policy in named data networking," IEEE Access, vol. 6, pp. 33702-33717, 2018.

[19] Y. Xu, Y. Li, S. Ci, T. Lin, and F. Chen, "Distributed caching via rewarding: an incentive caching model for ICN," in Proceedings of the GLOBECOM 2017-2017 IEEE Global Communications Conference, pp. 1-6, Singapore, December 2017.

[20] S. DiBenedetto, C. Papadopoulos, and D. Massey, "Routing policies in named data networking," in Proceedings of the ACM SIGCOMM Workshop on Information-Centric Networking, pp. 38-43, Association for Computing Machinery, New York, NY, USA, August 2011.

[21] N. Kamiyama, "CCN economics: can ISPs profit by introducing CCN?" in Proceedings of the 2014 10th International Conference on Network and Service Management (CNSM), pp. 242-247, IEEE, Rio de Janeiro, Brazil, November 2014.

[22] A. Araldo, D. Rossi, and F. Martignon, "Cost-aware caching: caching more (costly items) for less (ISPs operational expenditures)," IEEE Transactions on Parallel and Distributed Systems, vol. 27, no. 5, pp. 1316-1330, 2016.

[23] A. Ndikumana, K. Thar, T. M. Ho et al., "In-network caching for paid contents in content centric networking," in Proceedings of the GLOBECOM 2017-2017 IEEE Global Communications Conference, pp. 1-6, IEEE, Singapore, December 2017.

[24] M. Hajimirsadeghi, N. B. Mandayam, and A. Reznik, "Joint caching and pricing strategies for information centric networks," in Proceedings of the 2015 IEEE Global Communications Conference (GLOBECOM), pp. 1-6, IEEE, San Diego, CA, USA, December 2015.

[25] T.-M. Pham, "Analysis of ISP caching in information-centric networks," in Proceedings of the 2015 IEEE RIVF International Conference on Computing \& Communication TechnologiesResearch, Innovation, and Vision for Future (RIVF), pp. 151-156, IEEE, Can Tho, Vietnam, January 2015.

[26] B. Assila, A. Kobbane, and J. Ben-Othman, "Improving caching resource management: a pricing economic approach using Cournot, Bertrand, and Stackelberg game models," International Journal of Communication Systems, p. e4358, 2020, In press.

[27] P. Agyapong and M. Sirbu, "Economic incentives in information- centric networking: implications for protocol design and public policy," IEEE Communications Magazine, vol. 50, no. 12, pp. 18-26, 2012.

[28] M. Cha, H. Kwak, P. Rodriguez, Y.-Y. Ahn, and S. Moon, "I tube, you tube, everybody tubes: analyzing the world's largest user generated content video system," in Proceedings of the 7th ACM SIGCOMM Conference on Internet Measurement, pp. 1-14, ACM, San Diego, CA, USA, October 2007.

[29] C. Fricker, P. Robert, J. Roberts, and N. Sbihi, "Impact of traffic mix on caching performance in a content-centric network," in Proceedings of the Computer Communications Workshops (INFOCOM WKSHPS), pp. 310-315, IEEE, Orlando, FL, USA, March 2012.

[30] S. Vanichpun and A. M. Makowski, "The output of a cache under the independent reference model: where did the locality of reference go?" ACM SIGMETRICS Performance Evaluation Review, vol. 32, no. 1, pp. 295-306, 2004. 
[31] H. Garmani, D. Ait Omar, M. El Amrani, M. Baslam, and M. Jourhmane, "Analysis of a dynamics duopoly game with two content providers," Chaos, Solitons \& Fractals, vol. 131, Article ID 109466, 2020.

[32] H. Garmani, D. Ait Omar, M. El Amrani, M. Baslam, and M. Jourhmane, "Towards a predictive analysis of UAV-based flying base station decisions," International Journal of Business Data Communications and Networking, vol. 16, no. 2, pp. 20-52, 2020.

[33] H. Garmani, D. A. Omar, M. El Amrani, M. Baslam, and M. Jourhmane, "A dynamic duopoly game with content providers bounded rationality," International Journal of Bifurcation and Chaos, vol. 30, no. 7, Article ID 2050095, 2020.

[34] E. Altman, A. Legout, and Y. Xu, "Network non-neutrality debate: an economic analysis," in Proceedings of the International Conference on Research in Networking, pp. 68-81, Springer, Valencia, Spain, May 2011.

[35] H. Garmani, M. Baslam, and M. Jourhmane, "Analysis of competition between CPs fronting advertisers," International Journal of Advanced Science and Technology, vol. 117, pp. 53-66, 2018.

[36] H. Garmani, M. El Amrani, M. Baslam, R. El Ayachi, and M. Jourhmane, “A Stackelberg game-based approach for interactions among Internet service providers and content providers," NETNOMICS: Economic Research and Electronic Networking, vol. 20, no. 2-3, pp. 101-128, 2019.

[37] M. Outanoute, H. Garmani, M. Baslam, R. El Ayachi, and B. Bouikhalene, "A non-cooperative game analysis of competition between content providers in the internet market:," International Journal of Business Data Communications and Networking, vol. 15, no. 1, pp. 88-104, 2019.

[38] S. Lasaulce, M. Debbah, and E. Altman, "Methodologies for analyzing equilibria in wireless games," IEEE Signal Processing Magazine, vol. 26, no. 5, pp. 41-52, 2009.

[39] L. Guijarro, V. Pla, J. R. Vidal, and J. Martinez-Bauset, "Analysis of price competition under peering and transit agreements in internet service provision to peer-to-peer users," in Proceedings of the 2011 IEEE Consumer Communications and Networking Conference (CCNC), pp. 1145-1149, Las Vegas, NV, USA, January 2011.

[40] J. B. Rosen, "Existence and uniqueness of equilibrium points for concave N-person games," Econometrica, vol. 33, no. 3, p. 520, 1965. 